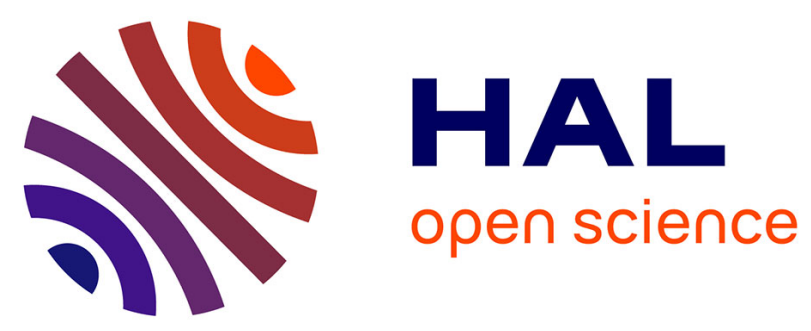

\title{
Modified Constitutive Relation Error for field identification : theoretical and experimental assessments on fiber orientation identification in a composite material
}

Renaud Ferrier, Aldo Cocchi, Christian Hochard

\section{To cite this version:}

Renaud Ferrier, Aldo Cocchi, Christian Hochard. Modified Constitutive Relation Error for field identification: theoretical and experimental assessments on fiber orientation identification in a composite material. International Journal for Numerical Methods in Engineering, 2021, 10.1002/nme.6842 . hal-03363459

\author{
HAL Id: hal-03363459 \\ https://hal.science/hal-03363459
}

Submitted on 4 Oct 2021

HAL is a multi-disciplinary open access archive for the deposit and dissemination of scientific research documents, whether they are published or not. The documents may come from teaching and research institutions in France or abroad, or from public or private research centers.
L'archive ouverte pluridisciplinaire HAL, est destinée au dépôt et à la diffusion de documents scientifiques de niveau recherche, publiés ou non, émanant des établissements d'enseignement et de recherche français ou étrangers, des laboratoires publics ou privés. 


\section{Modified Constitutive Relation Error for field identification :}

\section{theoretical and experimental assessments on fiber orientation}

\section{identification in a composite material}

\author{
${ }^{1}$ LMA, Aix-Marseille Université, Marseille, \\ France \\ Correspondence \\ *Renaud Ferrier. Email: \\ ferrier@1ma-cnrs-mrs.fr \\ Present Address \\ 4 impasse Nikola Tesla, 13013 Marseille, \\ France
}

Renaud Ferrier* | Aldo Cocchi | Christian Hochard

\begin{abstract}
Summary
This study addresses the application of the modified Constitutive Relation Error to field identification problems in the framework of elastostatics. We show how relevant is the addition of a gradient-penalizing regularization term (in norm $L^{1}$ or $L^{2}$ ), and emphasize the role played by unreliable boundary conditions. This leads to the proposition of a method using two parameters, for which automatic determination is addressed. All theoretical assessments are illustrated on experimental data. The test-problem consists in the identification of the heterogeneous fiber-orientation in a woven fabric composite from a unique quasi-static tensile test with digital image correlation.
\end{abstract}

\section{KEYWORDS:}

field identification, constitutive relation error, regularization, boundary conditions

\section{1 | INTRODUCTION}

Inverse field identification problems arise in many practical applications. For example in defect imaging, when we want to identify a zone where a given material parameter is far from the expected value. Another example is that of a sample in which the medium is heterogeneous because of the production process. In such a case, the knowledge of the material parameter field may be very approximate, which makes further numerical computations unreliable.

In this context, we suppose that an experimental test has been carried out to map a parameter's distribution by using partial measurements of the sample's response to a known excitation. In the experimental example of this article, the parameter to identify is the orientation of fibers in a composite, the excitation is a quasi-static tension load and the measurement is obtained by 
digital image correlation (DIC). This particular problem has been chosen because it is possible to obtain a reference orientation field by simple observation, and thus to evaluate the performance of the identification process.

The most straightforward identification method consists in finding the field of parameters that minimizes the difference between the experimental measurement and the same measurement simulated with a direct computation. This method is called PDE-constrained least-square minimization, and can also be found under the name Finite Element Method Update (FEMU). It is presented, among others, in ${ }^{1}$. In this study, we will use the modified Constitutive Relation Error (m-CRE), that has many similarities with least-square minimization.

The m-CRE method (also sometimes referred as MECE for Modified Error in Constitutive Equation) has been originally applied to identification under free vibration in ${ }^{2}$. Since then, it has been successfully applied on many different inverse problems, among which we can mention the identification of material parameters in the framework of transient dynamics ${ }^{\sqrt[3]{4}}$, identification in static elasticity ${ }^{5}$ and defect identification in composites under compressive load, leading to buckling 6 .

The iterative identification methods studied in this paper require to solve several forward problems. As a consequence, the quality of the identification is expected to depend on the validity of the hypotheses made on these forward problems, and in particular, the Boundary Conditions (BC). $\mathrm{In}^{[5}$ and ${ }^{7}$, the authors show how to use respectively m-CRE and least-square methods without having to feed the model with unreliable BC. They show that the quality of the identification is significantly better by this means.

Given that parameter identification problems are very often prone to instability, the use of regularization methods is an essential tool to obtain a solution that makes physical meaning. More precisely, the cost-function tends to be unable to discriminate different candidate-solutions, and the regularization consists in choosing the right solution among them. Of course, the meaning of right is a complex issue that must be defined for each particular application. Such regularization methods include for example the Truncated (Generalized) SVD $\stackrel{8}{\text {, }}$, or the Tikhonov method, of which one of the first occurrences can be found in ${ }^{9}$. Another approach, often used in the context of Constitutive Relation Error is to limit the number of iterations of the iterative algorithm. This is used for example in ${ }^{6}$.

In a first part, we present the test-problem, namely the identification of an heterogeneous field of fibers orientations in a composite, as well as the experimental setting. The second part is dedicated to the least-square minimization method and the m-CRE method. We recall how it is possible to ignore unreliable boundary conditions with the m-CRE method. The third part presents the minimization strategy, as well as the regularization procedure. Two different gradient-penalizing terms are presented, along with their theoretical pros and cons. The last part is dedicated to the numerical evaluation of the different proposed regularization strategies, as well as the different variants of the method. 


\section{2 | PRESENTATION OF THE APPLICATION PROBLEM}

Let us consider a part manufactured with a woven fabric-reinforced composite. During the forming process, before the polymerization, the part can be subjected to forming stresses which change the warp and weft orientation. This leads to an heterogeneous field of fibers orientation, that will be fixed during polymerization. This problem is for example studied in $\frac{10}{10} \operatorname{In} \frac{11}{\text {, the authors }}$ show how to carry on a numerical structure computation from the knowledge of this field of orientations.

In Section 2.3, we show that this field can be determined through a simple observation of the sample. The resulting field will be referred to as the reference orientation, and it will be used to evaluate the accuracy of the inverse procedure that consists in identifying these angles from the knowledge of the displacement field on the sample during a tensile test that is carried out after the polymerization.

\section{1 | Obtention of the test specimen}

In our case, we consider a rectangular coupon manufactured with a twill woven carbon/epoxy composite and a $[+45]_{S}$ layup. Before the polymerization, white lines parallel to the warp and weft directions are drawn on the surface of the sample. This provides a reference measurement of the fiber orientation. Afterwards, the coupon is stretched in the longitudinal ( 0 deg $)$ direction and takes the deformed shape observed on Figure $1 \mathrm{a}$.

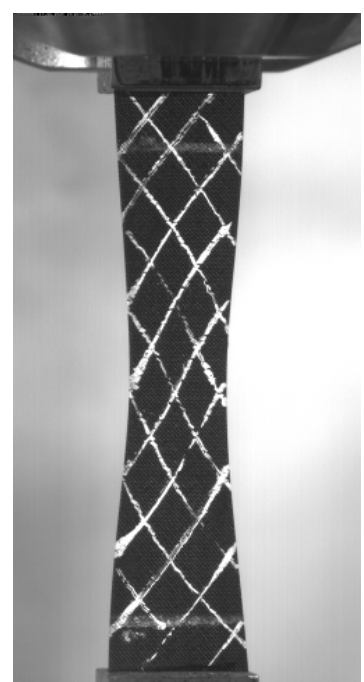

(a) Deformed specimen
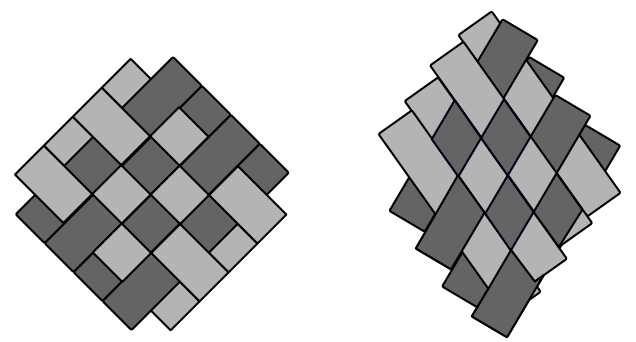

(b) Undeformed (left) and deformed (right) woven fabric composite

FIGURE 1 Tensile stress specimen and representation of the material deformation

Once the specimen has been deformed, it is polymerized. The result is a planar test specimen whose fiber orientation is heterogeneous. 


\section{2 | From specimen realization to inverse solution}

Once the heterogeneous test specimen has been obtained, a black and white speckle is painted on one of its faces and a tensile test until failure is performed. During the test, images of the specimen are recorded. These images are processed by the software GOM ARAMIS V5 $\frac{12}{12}$ in order to obtain a discretized displacement field at each time step corresponding to a picture.

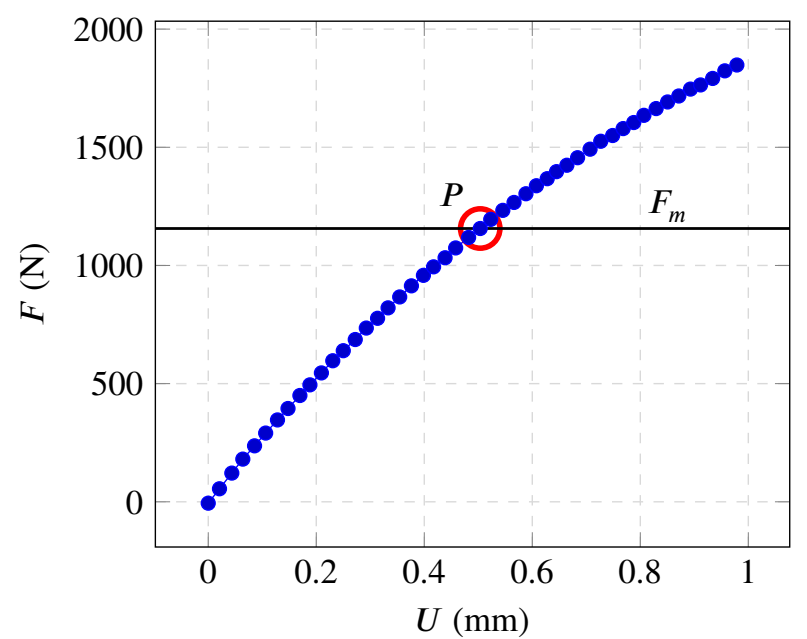

FIGURE 2 Macroscopical force-displacement evolution during the tensile test. $F_{m}$ is the minimal force for which non-linearity lead to more than $20 \%$ deviation from the linear behaviour. After this value, the behaviour is considered to be no longer linear enough for the inverse resolution

On the macroscopical force-displacement curve, displayed on Figure 2, one identifies the point $P$ until which the material behaviour is reasonably linear. For this value of displacement (here 0.504), the difference between the measured force and the force that would be obtained with a linear behaviour tangent at the origin is about $20 \%$. This point corresponds to a time step denoted by $t_{m}$. The macroscopical force at this time step is denoted by $F_{m}$. The knowledge of the measured displacement field for all time steps $t_{i} \leqslant t_{m}$ is useful to determine the uncertainty on the measure. For each point on the sample, we denote by $u_{x}(F)$ and $u_{y}(F)$ the function giving the horizontal or vertical displacement from the macroscopical force $F$. The nominal shape of $u_{x, y}$ is denoted by $u_{x, y}^{0}$ and is linear, and the uncertainty is computed as the deviation from this line by writing: $u_{x, y}(F)=u_{x, y}^{0}(F)+n$. Where the noise $n$ follows a Gaussian distribution which standard deviation is supposed to respect: $\sigma(F)=a F+b$. The parameters $a$ and $b$ are fitted independently for each point in space, and finally, the standard deviation of the measurement is $\sigma\left(F_{m}\right)$

It should be mentioned that the software GOM ARAMIS V5, like most Digital Image Correlation softwares, can estimate the uncertainty on the performed measurement. However, this estimation requires to perform a calibration operation, which has not been done in our case. What is more, our estimation of the standard deviation is expected to be more precise as it takes into account both measurement noise and errors due to the non-linearities (while the model used for the inversion is linear). 
Figure 3 recapitulates the whole protocol that has been followed to obtain the specimen, measure the reference fiber orientation and identify those orientations from a tensile test.

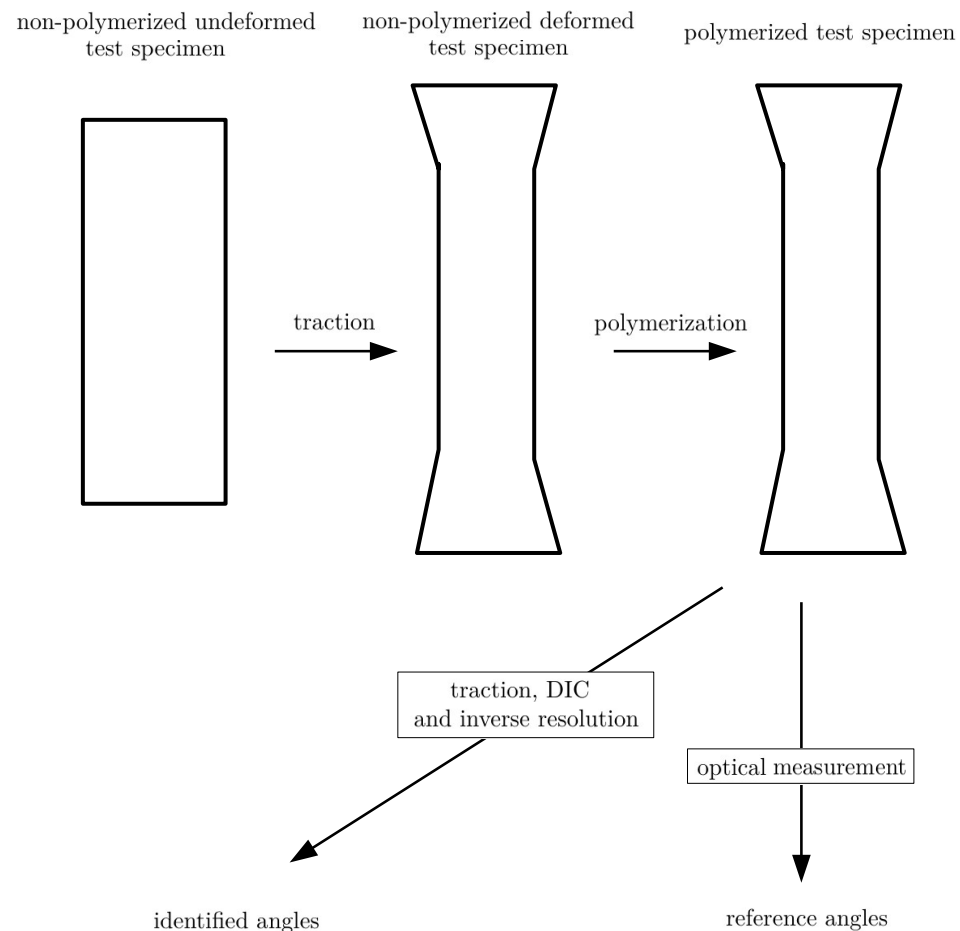

FIGURE 3 Recapitulation of different steps of the process

As the sample is heated during polymerization, it is reasonable to think that thermal residual stresses might be present in the sample at the end of the manufacturing process. However, the identification is only performed on the linear part of the post-polymerization tension curve, as a consequence these internal stresses do not impact the results.

\section{3 | Measurement of the fibers orientation}

The reinforcement of the composite specimen is composed of warp and weft fibers. We define two angles, $\theta_{1}$ and $\theta_{2}$, as shown on Figure 5 that parametrize respectively the orientation of the warp and of the weft.

For modelling purposes, the three dimensional woven can be assimilated to an assembly of two unidirectional virtual composites plies. The orientation angle of the fibres of these unidirectional plies can be measured from the white lines drawn on the specimen surface (Figure 1a). The cartography of these angles is given on Figure 4 It should be noted that the horizontal axis 
on Figure 4 is mirrored respect to the one on Figure 1a because the sample has been reverted in the machine before proceeding with the tensile test in order to present to the camera the side covered with a speckle.

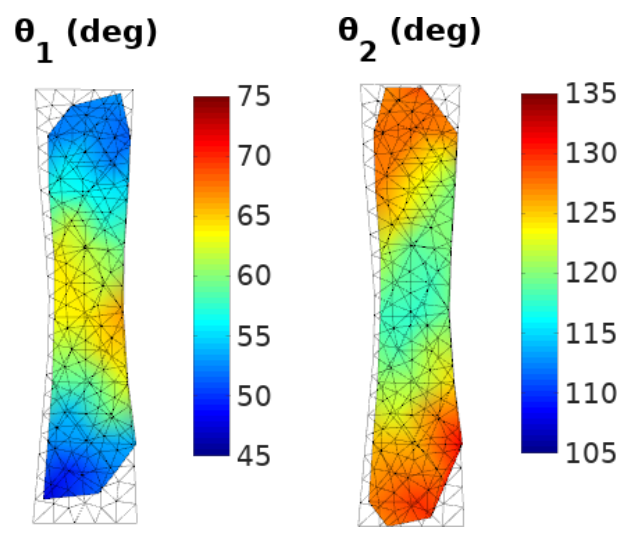

FIGURE 4 Measured angles

This map has a coarse spatial resolution, but it will however be used as a reference data to evaluate the accuracy of the inverse computation.

\section{4 | Properties of the material as a function of the orientations}

We determine the Hooke's tensor of the homogenized bidimensional material in function of the two angles. Let $H_{t o t}$ denote the Hooke's tensor in the specimen's coordinate system. $H_{1}$ and $H_{2}$ are respectively the warp's and the weft's Hooke's tensors.

$$
H_{t o t}=H_{1}+H_{2}
$$

With :

$$
H_{i}=e_{i} P_{i}^{-1} H_{0} P_{i}^{-T}
$$

Where $e_{i}, H_{0}$ and $P_{i}^{-1}$ are defined below.

$e_{i}$ is the thickness of the unidirectional virtual ply, that depends on $\theta_{1}$ and $\theta_{2}$. Let us consider the red polygon of Figure 5 If we make the approximation that the fields $\theta_{1}$ and $\theta_{2}$ are homogeneous inside this polygon, its shape is a rhombus. Let us make the hypothesis that the contact between warp and weft ensures that the fibers can not translate on the edges of this rhombus, but only rotate. Therefore, the volume of material inside the rhombus is the same for all values of $\theta_{1}$ and $\theta_{2}$. Moreover, this volume is equal to the surface of the rhombus multiplied by the thickness. 


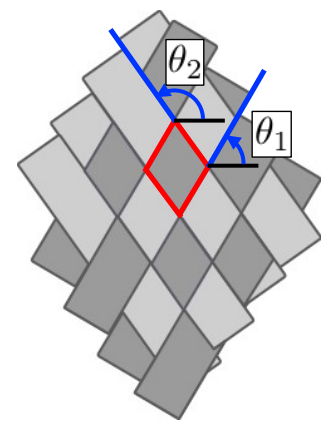

FIGURE 5 Parametrization of the deformed material

The surface of the rhombus equals $a^{2} \sin \left(\theta_{2}-\theta_{1}\right)$, where $a$ is the length of one of its sides. In the undeformed case, when $\theta_{1}=\frac{\pi}{4}$ and $\theta_{2}=\frac{3 \pi}{4}$, the rhombus is a square and its surface is $a^{2}$. Therefore, by conservation of volume, the thickness of the unidirectional virtual ply is equal to :

$$
e_{1}=e_{2}=e=\frac{e_{0}}{\sin \left(\theta_{2}-\theta_{1}\right)}
$$

$e_{0}$ is the thickness of an undeformed unidirectional virtual ply.

At this stage, one can compute a predicted value of the ratio between maximal and minimal thickness on the sample from the measured angles of Figure 4 . This ratio is equal to $\frac{\sin \left(\theta_{2}^{\max }-\theta_{1}^{\max }\right)}{\sin \left(\theta_{2}^{\min }-\theta_{1}^{\min }\right)}=1.19$. Alternatively, this ratio can also be measured on the specimen with a caliper resulting in a value of 1.22. These results show a reasonable agreement and this thickness information could probably be used somehow as prior information for the inverse problem. However, this aspect has not been investigated in this work.

We represent the stress tensor with Voigt notation:

$$
\underline{\underline{\sigma}}=\left(\begin{array}{ccc}
\sigma_{11} & \sigma_{12} & 0 \\
\sigma_{12} & \sigma_{22} & 0 \\
0 & 0 & 0
\end{array}\right) \leadsto \underline{\sigma}=\left(\begin{array}{c}
\sigma_{11} \\
\sigma_{22} \\
\sigma_{12}
\end{array}\right)
$$

In this context, one can compute the transformation matrix that is applied to $\underline{\sigma}$ to write the rotation of $\underline{\underline{\sigma}}$. This transformation matrix is denoted by $P_{i}^{-1}$ (because $P_{i}$ is used to describe the rotation of $\underline{\underline{\varepsilon}}$ ). This operation is explained for example in $\underline{[13}$. The expression of $P_{i}^{-1}$ is:

$$
P_{i}^{-1}=\left(\begin{array}{ccc}
\cos ^{2}\left(\theta_{i}\right) & \sin ^{2}\left(\theta_{i}\right) & -2 \sin \left(\theta_{i}\right) \cos \left(\theta_{i}\right) \\
\sin ^{2}\left(\theta_{i}\right) & \cos ^{2}\left(\theta_{i}\right) & 2 \sin \left(\theta_{i}\right) \cos \left(\theta_{i}\right) \\
\sin \left(\theta_{i}\right) \cos \left(\theta_{i}\right) & -\sin \left(\theta_{i}\right) \cos \left(\theta_{i}\right) & \cos ^{2}\left(\theta_{i}\right)-\sin ^{2}\left(\theta_{i}\right)
\end{array}\right)
$$


One computes the derivatives :

$$
\begin{aligned}
\frac{\partial H_{i}}{\partial \theta_{i}} & =\frac{\partial e_{i}}{\partial \theta_{i}} P_{i}^{-1} H_{0} P_{i}^{-T}+e_{i} \frac{\partial P_{i}^{-1}}{\partial \theta_{i}} H_{0} P_{i}^{-T}+e_{i} P_{i}^{-1} H_{0}\left[\frac{\partial P_{i}^{-1}}{\partial \theta_{i}}\right]^{T} \\
\frac{\partial e}{\partial \theta_{2}} & =-\frac{\partial e}{\partial \theta_{1}}=\frac{\cos \left(\theta_{2}-\theta_{1}\right)}{\sin ^{2}\left(\theta_{2}-\theta_{1}\right)} \\
\frac{\partial P_{i}^{-1}}{\partial \theta_{i}} & =\left(\begin{array}{ccc}
-2 \cos \left(\theta_{i}\right) \sin \left(\theta_{i}\right) & 2 \cos \left(\theta_{i}\right) \sin \left(\theta_{i}\right) & -2 \cos ^{2}\left(\theta_{i}\right)+2 \sin ^{2}\left(\theta_{i}\right) \\
2 \cos \left(\theta_{i}\right) \sin \left(\theta_{i}\right) & -2 \cos \left(\theta_{i}\right) \sin \left(\theta_{i}\right) & 2 \cos ^{2}\left(\theta_{i}\right)-2 \sin ^{2}\left(\theta_{i}\right) \\
\cos ^{2}\left(\theta_{i}\right)-\sin ^{2}\left(\theta_{i}\right) & -\cos ^{2}\left(\theta_{i}\right)+\sin ^{2}\left(\theta_{i}\right) & -4 \cos \left(\theta_{i}\right) \sin \left(\theta_{i}\right)
\end{array}\right)
\end{aligned}
$$

It is worth noting that $H_{\text {tot }}$ is $\pi$ periodic with respect to $\theta_{1}$ and $\theta_{2}$. As a consequence, the angles are not sought in $\mathbb{R}$, but in $[0, \pi]$. This has no consequence provided, during the execution of the algorithm, the values taken by $\theta_{i}$ stay far form 0 and $\pi$.

One important point for the reliability of the identification is that the knowledge of the material parameter must itself be reliable. This is of course not the case. One could propose to identify these parameters during a prior test on a part for which the fiber orientation is known and homogeneous. In the present case, we prefer to add the 4 material parameters, supposed to be uniform, to the unknowns. In order not to spoil the conditioning of the Hessian, the unknowns that are added in practice are normalized by the initial values of the material parameters.

The Hooke's tensor $H_{0}$ and its derivatives with respect to the material parameters are computed:

$$
H_{0}=S_{0}^{-1}=\left(\begin{array}{ccc}
\frac{1}{E_{1}} & -\frac{v_{12}}{E_{1}} & 0 \\
-\frac{v_{12}}{E_{1}} & \frac{1}{E_{2}} & 0 \\
0 & 0 & \frac{1}{G_{12}}
\end{array}\right)^{-1}=\left(\begin{array}{ccc}
\frac{E_{1}^{2}}{E_{1}-v_{12}^{2} E_{2}} & \frac{v_{12} E_{1} E_{2}}{E_{1}-v_{12}^{2} E_{2}} & 0 \\
\frac{v_{12} E_{1} E_{2}}{E_{1}-v_{12}^{2} E_{2}} & \frac{E_{1} E_{2}}{E_{1}-v_{12}^{2} E_{2}} & 0 \\
0 & 0 & G_{12}
\end{array}\right)
$$




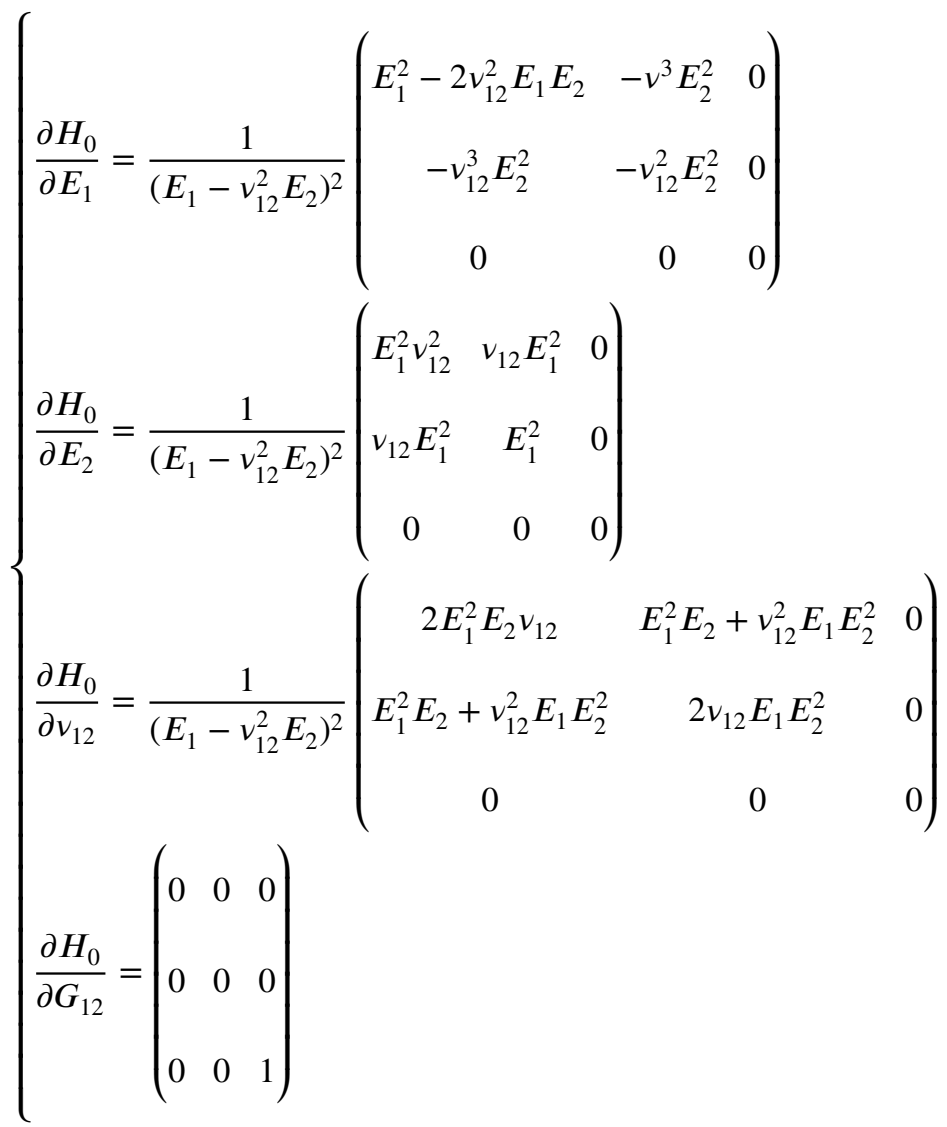

Practical tests show that the sensibility of the m-CRE cost function with respect to the parameters $E_{2}$ and $v_{12}$ is too low to ensure a reliable identification. As a consequence, only $E_{1}$ and $G_{12}$ are identified.

The initial values taken for the material parameters are reported in 99 .

$$
\left\{\begin{array}{l}
E_{1}=150 G P a \\
E_{2}=10 G P a \\
v_{12}=0.3 \\
G_{12}=3.8 \mathrm{GPa}
\end{array}\right.
$$

\section{3 | FIELD IDENTIFICATION METHODS}

The problem of interest is the identification of two (discretized) fields of parameters from displacement measurements. This results in numerous unknowns, that can be gathered in a vectorial unknown, denoted by $\theta$. In the framework of linear elastostatics, the discretized direct problem consists in computing the displacement field on a discretized domain $\Omega_{h}$ from the 
knowledge of boundary conditions and material parameters $\theta$. The problem is discretized with the Finite Element Method (FEM), and $\theta$ only influences the stiffness matrix, $\breve{K}(\theta)$. The direct problem can then be written under the following form:

$$
u=\underset{\substack{x \\ C x=\alpha}}{\arg \min } \frac{1}{2} x^{T} \breve{K}(\theta) x-x^{T} \breve{f}
$$

$u$ is the unknown displacement field on $\Omega_{h}, C x=\alpha$ is a set of linear constraints that impose, among others, the discretized Dirichlet Boundary Conditions (BC). One supposes that $C$ has full row rank, which means that all constraints in $C x=\alpha$ are linearly independent. As a consequence, $C C^{T}$ is an invertible matrix.

The methods will be presented formally in the case when the constraints are satisfied by substitution, but they are of course applicable with any constrained minimization method. One can show that Problem $(10)$ is equivalent to Problem $(11)$ :

$$
\begin{aligned}
u & =\underset{x}{\arg \min } \frac{1}{2} x^{T} K(\theta) x-x^{T} f \\
K(\theta) & =\left(\mathcal{I}_{d}-C^{T}\left(C C^{T}\right)^{-1} C\right) \breve{K}(\theta)\left(\mathcal{I}_{d}-C^{T}\left(C C^{T}\right)^{-1} C\right)+\bar{K} C^{T}\left(C C^{T}\right)^{-1} C \\
f & =\left(\mathcal{I}_{d}-C^{T}\left(C C^{T}\right)^{-1} C\right)\left(\breve{f}-\breve{K}(\theta) C^{T}\left(C C^{T}\right)^{-1} \alpha\right)+\bar{K} C^{T}\left(C C^{T}\right)^{-1} \alpha
\end{aligned}
$$

$\mathcal{I}_{d}$ is the identity matrix of the same size as $\breve{K}(\theta)$, and $\bar{K}$ is a scalar parameter, determined as an estimation of the spectral radius of $\breve{K}(\theta)$. It prevents the resulting system from being ill-conditioned in the case when $\breve{K}(\theta)$ involves large moduli. In practice, it is often not necessary to take this parameter different to 1 .

Problem (11) reduces to the linear problem (12). This approach has the notational advantage that the solution of this problem is exactly $u$, and not a restriction of it, nor a vector containing $u$ and Lagrange multipliers.

$$
K(\theta) u=f
$$

The inverse problem consists in computing $\theta$ from the knowledge of a measured displacement, denoted by $u_{m}$. This measurement was done on a particular grid, driven by the measurement method, which has no reason to coincide with the FE mesh. For that reason, one introduces $\Pi$, the observation operator such that $\Pi u$ is defined on the same grid as $u_{m}$. This observation operator introduces an interpolation error that will be neglected in this work.

What is more, the question of the discretization of $\theta$ is crucial for the well-posedness of the mathematical problem because the more unknowns there are, the less stable the solution is. For the sake of simplicity, in this study, it has been decided to use the FE mesh to discretize $\theta$. This results in the inverse problem being very unstable and most likely having no unique solution. To solve this, and in order to make the solution mesh-independent, we introduce a regularizing (semi-) norm, denoted by $N(\theta)$, that is used to stabilize the solution. The weight of this term is tuned by a regularization parameter $\mu$. 


\section{1 | Least-square minimization}

One writes the following minimization problem, where the FE-discretized $\operatorname{PDE} K(\theta) u=f$ is added as a constraint:

$$
\begin{gathered}
\min _{\substack{\theta, u \\
K(\theta) u=f}} \varphi(\theta, u) \\
\varphi(\theta, u)=\frac{1}{2}\left(\Pi u-u_{m}\right)^{T}\left(\Pi u-u_{m}\right)+\mu N(\theta)
\end{gathered}
$$

The problem (13) is equivalent to the search of the saddle point of the following Lagrangian:

$$
\phi(\theta, u, \lambda)=\frac{1}{2}\left(\Pi u-u_{m}\right)^{T}\left(\Pi u-u_{m}\right)+\mu N(\theta)+\lambda^{T}(K(\theta) u-f)
$$

One can express the gradients of $\phi$ :

$$
\left\{\begin{array}{l}
\nabla_{u} \phi=\Pi^{T}\left(\Pi u-u_{m}\right)+K(\theta) \lambda \\
\nabla_{\lambda} \phi=K(\theta) u-f \\
\nabla_{\theta} \phi=\mu \nabla N(\theta)+\lambda^{T} \nabla K(\theta) u
\end{array}\right.
$$

For solving (13), we use the BFGS method, as detailed in Section 4.1 In the present case, the BFGS method requires to find $u$ and $\lambda$ that make respectively $\nabla_{\lambda} \phi$ and $\nabla_{u} \phi$ vanish. Therefore we solve the following direct and adjoint problems at each BFGS iteration:

$$
\left\{\begin{array}{l}
K(\theta) u=f \\
K(\theta) \lambda=\Pi^{T}\left(u_{m}-\Pi u\right)
\end{array}\right.
$$

\section{2 | Modified Constitutive Relation Error}

In this section, we present a variant of the method of modified Constitutive Relation Error, in the case where there are available and reliable Boundary Conditions on the entire boundary of the studied domain. This variant is called MCRE-I in ${ }^{5}$.

Let us introduce two displacement fields, that are defined on the FE mesh. $v$ respects the global equilibrium, which means that it is the unique solution to the direct problem $K(\theta) v=f$. As $K(\theta)$ is built as described in Equation (11), $C v=\alpha$ is ensured. $u$ is supposed to be equal to the measured displacement, which means $\Pi u=u_{m}$, but the modified CRE method relaxes this condition by adding term penalizing the norm of $\Pi u-u_{m}$.

We choose to impose that $u$ respects the Dirichlet BCs of the direct problem, ie. $C u=\alpha$. This assumption makes sure that $u$ and $v$ are in the same space. 
The minimization problem is the following:

$$
\min _{\substack{\theta, u, v \\ C u=\alpha, K(\theta) v=f}} \frac{1}{2}(u-v)^{T} \breve{K}(\theta)(u-v)+\frac{r}{2}\left(\Pi u-u_{m}\right)^{T}\left(\Pi u-u_{m}\right)+\mu r N(\theta)
$$

We remark that $C(u-v)=0$, and given the expression of $K(\theta)$ in Equation 11 , we have $(u-v)^{T} \breve{K}(\theta)(u-v)=(u-v)^{T} K(\theta)(u-v)$, which allows to replace this term in (17), in order to obtain the following minimization problem, that does not use $\breve{K}(\theta)$ anymore.

$$
\min _{\substack{\theta, u, v \\ C u=\alpha, K(\theta) v=f}} \frac{1}{2}(u-v)^{T} K(\theta)(u-v)+\frac{r}{2}\left(\Pi u-u_{m}\right)^{T}\left(\Pi u-u_{m}\right)+\mu r N(\theta)
$$

In this method, two parameters have been introduced. The weighting parameter $r$ tunes the importance given to the measurements, and the regularization parameter $\mu$ penalizes the fields $\theta$ that have a high norm $N(\theta)$. Most authors use $r$ as a regularization parameter, as in 14 , where this parameter is changed at each iteration. In this context, $r$ can be determined with the L-curve or Morozov methods, that are exposed in Section 5.4.2

The term $N(\theta)$, while being very classical for the PDE-constrained least-square minimization method, is usually not used in m-CRE method. To the author's knowledge, the only occurrence of such a term in literature is very recent, in 15 , where a damage field is identified. One of the objectives of this study is to show the benefit of using this term for identifying smooth fields.

Note that the regularizing term is multiplied by the parameter $r$ in order to be consistent with the cost-function used by the least-square method. The proposed cost-function is then a sum of a term proportional to $\varphi$ from $(13)$ and the new Constitutive Relation Error term.

As previously, one can introduce the following Lagrangian, which saddle-point gives the solution to the problem:

$$
\phi(\theta, u, v, \lambda)=\frac{1}{2}(u-v)^{T} K(\theta)(u-v)+\frac{r}{2}\left(\Pi u-u_{m}\right)^{T}\left(\Pi u-u_{m}\right)+\mu r N(\theta)+\lambda^{T}(K(\theta) v-f)
$$

The gradients are:

$$
\left\{\begin{array}{l}
\nabla_{u} \phi=K(\theta) u-K(\theta) v+r \Pi^{T} \Pi u-r \Pi^{T} u_{m} \\
\nabla_{v} \phi=K(\theta) v-K(\theta) u+K(\theta) \lambda \\
\nabla_{\lambda} \phi=K(\theta) v-f \\
\nabla_{\theta} \phi=\frac{1}{2}(u-v)^{T} \nabla K(\theta)(u-v)+\mu r \nabla N(\theta)+\lambda^{T} \nabla K(\theta) v
\end{array}\right.
$$


Similarly to what was done in Section 3.1, one determines $u, v$ and $\lambda$ by writing $\nabla_{u} \phi=0, \nabla_{v} \phi=0$ and $\nabla_{\lambda} \phi=0$. This leads to solve the following uncoupled problems:

$$
\left\{\begin{array}{l}
K(\theta) v=f \\
\left(K(\theta)+r \Pi^{T} \Pi\right) u=f+r \Pi^{T} u_{m} \\
\lambda=u-v
\end{array}\right.
$$

Two linear problems have to be solved. The computational cost is the same as in the least-square method of Section 3.1 at one condition: the observation operator $\Pi$ has to be sparse.

One can finally notice that the least-square minimization method is a limit case of modified Constitutive Relation Error. Let us suppose that $r=o(\kappa)$, where $\kappa$ is the ratio between the spectral radii of $K$ and $\Pi^{T} \Pi$. We also suppose that $\mu=O(p)$, where $p$ is the ratio between the spectral radius of $\Pi^{T} \Pi$ and $G^{T} G$ (see Section 4.2). Schematically, this means that the terms $\frac{r}{2}\left(\Pi u-u_{m}\right)^{T}\left(\Pi u-u_{m}\right)+\mu r N(\theta)$ are much smaller than $\frac{1}{2}(u-v)^{T} K(\theta)(u-v)$ in $\phi$. In that case, as shown in $\frac{16}{\sqrt{6}}$, and as illustrated numerically in Section 5.2.3 the solution of the m-CRE methods tends towards the one of the least-square minimization method.

\section{3 m-CRE without boundary conditions on a part of the boundary}

As the measurement $u_{m}$ results from an experimental test, it is very likely that some of the BCs of this test are not well known. More specifically, for the case of a static tensile test, the displacement in the vicinity of the machine's jaws is unknown. As a consequence, we no longer take those BCs into account in the computation of $v$. This variant is called MCRE-II in ${ }^{5}$.

For the purpose of our application, we suppose in this part that all Dirichlet boundary conditions are ignored, which means that there is no condition of the type $C u=\alpha$, and $\breve{K}(\theta)=K(\theta)$.

The Degrees Of Freedom (DOF) subjected to unknown forces are denoted with a $\varnothing$ index while the other ones, for which an equilibrium relation can be written, are denoted with a 1 index.

One introduces the following decompositions for $K$ and $f$, and a prolongation by zero of $\lambda$, denoted by $\lambda_{2}$ :

$$
K(\theta)=\left(\begin{array}{cc}
K_{11}(\theta) & K_{1 \varnothing}(\theta) \\
K_{1 \varnothing}^{T}(\theta) & K_{\varnothing \varnothing}(\theta)
\end{array}\right)=\left(\begin{array}{c}
K_{1}(\theta) \\
K_{\varnothing}(\theta)
\end{array}\right) \quad f=\left(\begin{array}{c}
f_{1} \\
f_{\varnothing}
\end{array}\right) \quad \lambda_{2}=\left(\begin{array}{c}
\lambda \\
\lambda_{\varnothing}
\end{array}\right)
$$

where $\lambda_{\varnothing}$ is a null vector which size is the number of degrees of freedom with unknown force.

The known part of the equilibrium is:

$$
K_{1}(\theta) v=f_{1}
$$


The cost-function is slightly different from $[18]$ :

$$
\min _{\substack{\theta, u, v \\ K_{1}(\theta) v=f_{1}}} \frac{1}{2}(u-v)^{T} K(\theta)(u-v)+\frac{r}{2}\left(\Pi u-u_{m}\right)^{T}\left(\Pi u-u_{m}\right)+\mu r N(\theta)
$$

The Lagrangian is also slightly different from (19):

$$
\phi(\theta, u, v, \lambda)=\frac{1}{2}(u-v)^{T} K(\theta)(u-v)+\frac{r}{2}\left(\Pi u-u_{m}\right)^{T}\left(\Pi u-u_{m}\right)+\mu r N(\theta)+\lambda^{T}\left(K_{1}(\theta) v-f\right)
$$

And the gradients are:

$$
\left\{\begin{array}{l}
\nabla_{u} \phi=K(\theta) u-K(\theta) v+r \Pi^{T} \Pi u-r \Pi^{T} u_{m} \\
\nabla_{v} \phi=K(\theta) v-K(\theta) u+K_{1}^{T}(\theta) \lambda \\
\nabla_{\lambda} \phi=K_{1}(\theta) v-f_{1} \\
\nabla_{\theta} \phi=\frac{1}{2}(u-v)^{T} \nabla K(\theta)(u-v)+\mu r \nabla N(\theta)+\lambda^{T} \nabla K_{1}(\theta) u
\end{array}\right.
$$

Contrary to what happens in Section 3.2. one can not uncouple the three problems $\nabla_{u} \phi=0, \nabla_{v} \phi=0$ and $\nabla_{\lambda} \phi=0$.

$\nabla_{\lambda} \phi=0$ implies $K_{1}(\theta) v=f_{1}$ and $\nabla_{v} \phi=0$ gives:

$$
K(\theta)(u-v)=K_{1}^{T}(\theta) \lambda
$$

That can be restricted to the degrees of freedom where the force is known:

$$
K_{1}(\theta)(u-v)=K_{11}(\theta) \lambda
$$

We replace $K(\theta)(u-v)$ by $K_{1}^{T}(\theta) \lambda$ in $\nabla_{u} \phi=0$, which implies:

$$
K_{1}^{T}(\theta) \lambda=r \Pi^{T}\left(u_{m}-\Pi u\right)
$$

From equations 29 and 28 , one can write the system to determine $u$ and $\lambda$ :

$$
\left(\begin{array}{cc}
r \Pi^{T} \Pi & K_{1}^{T}(\theta) \\
K_{1}(\theta) & -K_{11}(\theta)
\end{array}\right)\left(\begin{array}{l}
u \\
\lambda
\end{array}\right)=\left(\begin{array}{c}
r \Pi^{T} u_{m} \\
f_{1}
\end{array}\right)
$$

The physical problem that is solved by the system (30) is similar to a prolongation problem, and consists in finding a field from measurements inside the domain and a PDE that governs the field, but without BCs. If some boundaries of the meshed domain, on which $u$ is defined, are far from any measurement point, this problem is ill-conditioned and a regularizing approach must be used $\left(\mathrm{se}^{\left({ }^{5}\right.}\right)$. In extreme cases, one could even propose to use methods dedicated to this type of problem, like the one 
introduced in $\frac{17}{17}$. However, in the cases that are studied here, we assume that there are always measurements close enough to the bounds of the domain to ensure that the problem (30) is well-conditioned and can be solved without any particular care.

From Equation [27, one gets $K(\theta)(u-v)=K(\theta) \lambda_{2}$. We remark that the term that actually appears in $\phi$ and its gradient is $K(\theta) v$, and the vector $v$ can be determined without any consideration for the kernel of $K(\theta)$ by a simple subtraction:

$$
v=u-\lambda_{2}
$$

From this equation, one can deduce:

$$
\left\{\begin{array}{l}
(u-v)^{T} K(\theta)(u-v)=\lambda_{2}^{T} K(\theta) \lambda_{2}=\lambda^{T} K_{11}(\theta) \lambda \\
(u-v)^{T} \nabla K(\theta)(u-v)=\lambda_{2}^{T} \nabla K(\theta) \lambda_{2}=\lambda^{T} \nabla K_{11}(\theta) \lambda
\end{array}\right.
$$

These equations lead to more practical and less CPU-costly expressions of the cost-function and its gradient.

\section{4 | MINIMIZATION AND REGULARIZATION}

The performance of an inversion method depends largely on the choice of the minimization method, as well as the regularizing approach.

\section{1 | Minimization method}

All methods presented in Section 3 consist in searching for a saddle-point of a function denoted by $\phi(\theta, u, v, \lambda)$. If we suppose that the saddle-point is unique, one can denote it by the notation argstat (the argument of the stationarity problem).

The saddle-point is a minimum in the direction $\theta$. One can then introduce a function $\psi$ of $\theta$ :

$$
\begin{aligned}
\psi(\theta) & =\phi(\theta, \tilde{u}(\theta), \tilde{v}(\theta), \tilde{\lambda}(\theta)) \\
\text { Where }\{\tilde{u}(\theta), \tilde{v}(\theta), \tilde{\lambda}(\theta)\} & =\underset{u, v, \lambda}{\operatorname{argstat}} \phi(\theta, u, v, \lambda)
\end{aligned}
$$

We will minimize $\psi$ with the BFGS method. This consists in an iterative method that requires, at each iteration $i$, to evaluate $\psi\left(\theta_{i}\right)$ and its gradient $\nabla \psi\left(\theta_{i}\right)$, which coincides with $\nabla_{\theta} \phi(\theta, \tilde{u}(\theta), \tilde{v}(\theta), \tilde{\lambda}(\theta))$, as shown in the following equation:

$$
\nabla \psi=\nabla_{\theta} \phi+\underbrace{\left[\nabla_{u} \phi\right]^{T}}_{=0} \nabla_{\theta} \tilde{u}+\underbrace{\left[\nabla_{v} \phi\right]^{T}}_{=0} \nabla_{\theta} \tilde{v}+\underbrace{\left[\nabla_{\lambda} \phi\right]^{T}}_{=0} \nabla_{\theta} \tilde{\lambda}=\nabla_{\theta} \phi
$$


For the Hessian of $\psi$, the BFGS method (see for example $\frac{18}{18}$ ) consists in approximating it by a matrix $\mathcal{H}_{i}$, that is obtained through an updating process that adds two new terms of rank 1 after each iteration.

$$
\begin{aligned}
y_{i} & =\nabla \psi\left(\theta_{i}\right)-\nabla \psi\left(\theta_{i-1}\right) \\
\delta_{i} & =\theta_{i}-\theta_{i-1} \\
\mathcal{H}_{i+1} & =\mathcal{H}_{i}+\frac{y_{i} y_{i}^{T}}{y_{i}^{T} \delta_{i}}-\frac{\mathcal{H}_{i} \delta_{i} \delta_{i}^{T} \mathcal{H}_{i}}{\delta_{i}^{T} \mathcal{H}_{i} \delta_{i}}
\end{aligned}
$$

$\mathcal{H}_{0}$ is initialized as a real parameter $\chi$ times the identity matrix $\mathcal{I}$. By this means, the first iteration of the method consists in a steepest descent step. $\chi$ has the dimension of an energy in our case where $\theta$ is dimensionless, but the choice of its value is not crucial as the step is tuned during the line-search procedure.

Newton's method is applied on the gradient of $\psi$, which results in each iterate to be written as follows:

$$
\tilde{\theta}_{i+1}=\theta_{i}-\mathcal{H}_{i}^{-1} \nabla \psi\left(\theta_{i}\right)
$$

When $\theta$ is updated in (36), we don't use the true Hessian, moreover, the cost-function can be arbitrarily far from a quadratic function. For these reasons, nothing ensures that $\psi\left(\tilde{\theta}_{i+1}\right)$ is actually lower than $\psi\left(\theta_{i}\right)$. This is why the following line-search procedure may be essential for the convergence of the algorithm.

The idea is to use the direction given by equation (36). One searches, along the ray $\left[\theta_{i}, \tilde{\theta}_{i+1}\right)$, the point $\theta_{i+1}$ that realizes the minimum of $\psi$. This can for example be done by dichotomy. in the present case, our algorithm is even simpler and consists in dividing the step by 2 until $\psi\left(\theta_{i+1}\right)<\psi\left(\theta_{i}\right)$. The advantage is that if $\tilde{\theta}_{i+1}$ satisfies this condition (which often occurs), the procedure does not require any more evaluation of $\psi$.

Let us emphasize that $\mathcal{H}$ is a full matrix. As a consequence, in the case where its size is big (one or a few values per Gauss point of a FE mesh), the inversion of the linear system may be numerically costly.

The BFGS method can converge towards any kind of stationary point of $\psi$. As a consequence, this procedure is a minimization method only if the cost-function is convex. In practice, it is not possible to ensure this, and one simply supposes that $\psi$ is locally convex in a given neighbourhood around its minimum, and that all iterates are in this neighbourhood. This is obviously not always true, that is why the choice of the initialization is important. In this study, two different initialization strategies are proposed in Section 4.2 that depend on the chosen regularizing term.

The m-CRE identification algorithm with the BFGS optimization method, and unreliable boundary conditions is summarized on Algorithm 1 


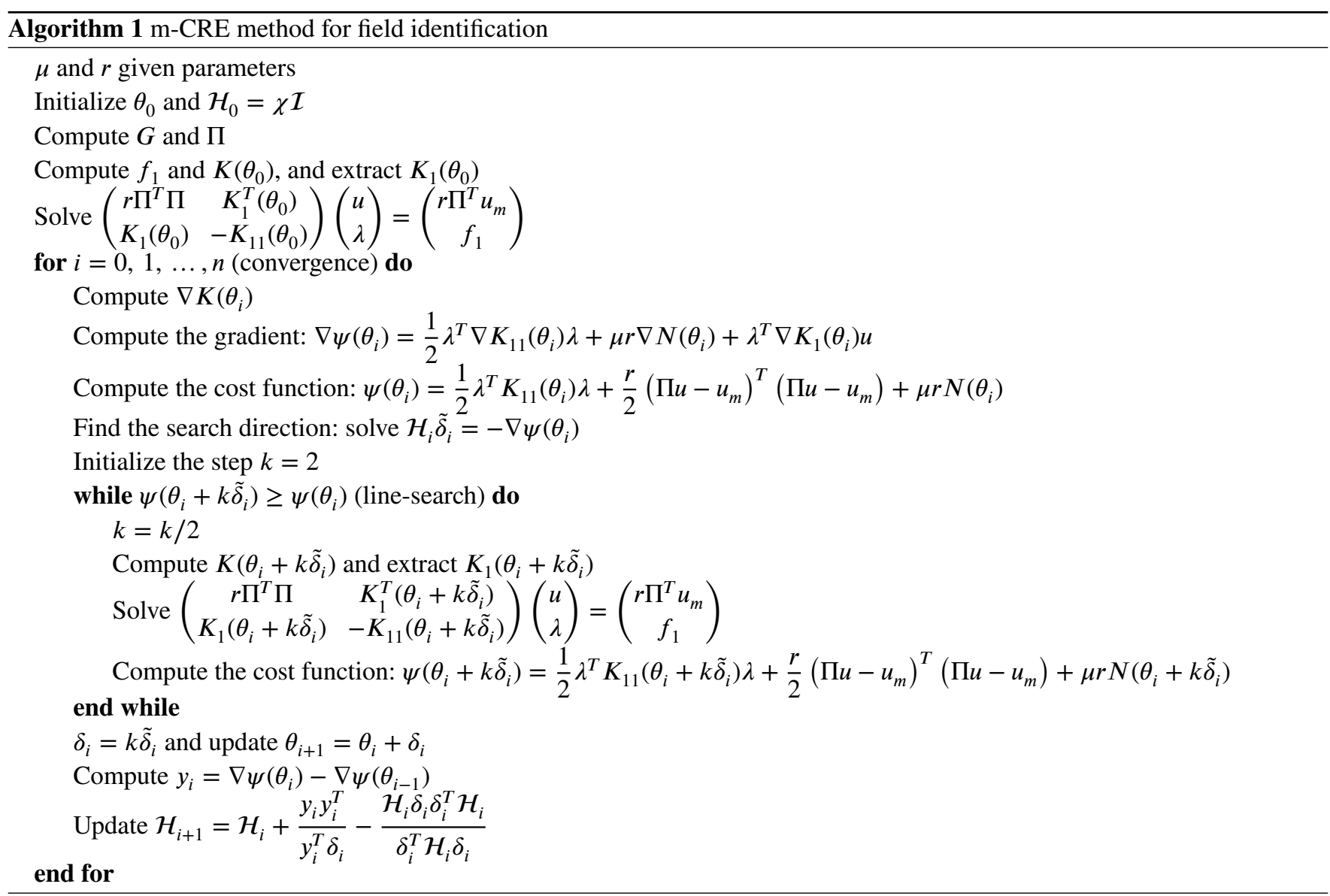

\section{2 | Regularizing norm}

The term $\mu N(\theta)$ is added to the cost-function in order to penalize the strong variations of $\theta$. For that purpose, we introduce $G$, the operator that gives the discrete gradient of $\theta$. In our implementation, $\theta$ is defined at the Gauss points of finite elements, the construction of $G$ is thus not straightforward. It was decided to build a Delaunay triangulation from the Gauss points, to eliminate triangles having the worst shape, and to interpolate $\theta$ on this new mesh in order to compute its gradient.

In what follows, two semi-norms of $\theta$, denoted by $N_{2}$ and $N_{1}$, are presented. They correspond respectively to the $L^{2}$ and the $L^{1}$ norms of $G \theta$.

\subsection{1 । $L^{2}$ norm of the gradient}

The most classically used regularizing term is the squared of the $L^{2}$ norm:

$$
N_{2}(\theta)=\frac{1}{2} \theta^{T} G^{T} G \theta
$$


The computation of its gradient leads to:

$$
\nabla_{\theta} N_{2}(\theta)=G^{T} G \theta
$$

When this norm is used, the unknown parameter vector $\theta$ can be initialized as a homogeneous field equal to (or rather not so far from) the expected mean of the parameter distribution.

\subsection{2 । $L^{1}$ norm of the gradient}

In the context of image restoration ${ }^{19}$ or compressed sensing ${ }^{20}$, many authors have proposed to use regularization terms that are based on the $L^{1}$ norm. This norm, along with the $L^{0}$ and other $L^{p}, 0<p<1$ norms, may be used to obtain a sparse representation of a set of unknowns. The review paper ${ }^{21}$ presents mathematical investigations and algorithms used in this framework.

To understand the potential benefit of using the $L^{1}$ norm of the gradient rather than the squared of its $L^{2}$ norm, one can compare, in the continuous unidirectional framework, the value of these two norms, denoted by $\|f\|_{p, \mathbb{R}}$, for the classical arctangent smoothed step function:

$$
\begin{aligned}
& k \in \mathbb{R}, \quad f: x \in \mathbb{R} \mapsto \arctan (k x) \\
& \|f\|_{2, \mathbb{R}}^{2}=\int_{-\infty}^{\infty}\left(f^{\prime}(x)\right)^{2} d x=k^{2} \frac{\pi}{2} \quad \text { and } \quad\|f\|_{1, \mathbb{R}}=\int_{-\infty}^{\infty}\left|f^{\prime}(x)\right| d x=\pi
\end{aligned}
$$

When $k$ is low, the function $f$ is smooth, while when $k$ tends towards $\infty$, it is known to approach a step function. One notices by the expressions of (39) that the $L^{2}$ norm of the gradient unsurprisingly increases with $k$, while the $L^{1}$ norm remains the same. This last norm only takes into account the total amount of absolute variation of the studied function, and not the rate of this variation. This is why it is often referred as the Total Variation term.

Penalizing the $L^{2}$ norm favours smooth variations (lower $k$ in our example) while penalizing the $L^{1}$ norm preserves sharp frontiers between quite homogeneous zones. It is very interesting if the parameter is suspected to obey to such a spatial distribution.

In the discrete framework, if $(G \theta)_{i}$ is the $i$-th term of the vector $G \theta$, one has:

$$
N_{1}(\theta)=\sum_{i}\left|(G \theta)_{i}\right|
$$

This norm has the inconvenient to be non-smooth and non-differentiable. As a consequence, the resolution of the regularized minimization problem requires to use dedicated methods, as proposed in ${ }^{19}$ or ${ }^{22}$. However, in our case, for simplicity, we 
prefer to use the more common BFGS algorithm. For this purpose, we propose to use a well-known smooth and differentiable approximation of $N_{1}(\theta)$, which is called $N_{1}^{\epsilon}(\theta)$ and defined on Equation 41].

$$
N_{1}^{\epsilon}(\theta)=\sum_{i} \sqrt{\left((G \theta)_{i}\right)^{2}+\epsilon}
$$

Figure 6 compares $|\cdot|$ and its smoothed version $|\cdot|^{\epsilon}=\sqrt{\cdot^{2}+\epsilon}$ with $\epsilon=10^{-2}$. For information, in the identification process of Section 5 , we use $\epsilon=10^{-10}$ and the values of $(G \theta)_{i}$ are around $10^{-5}$.

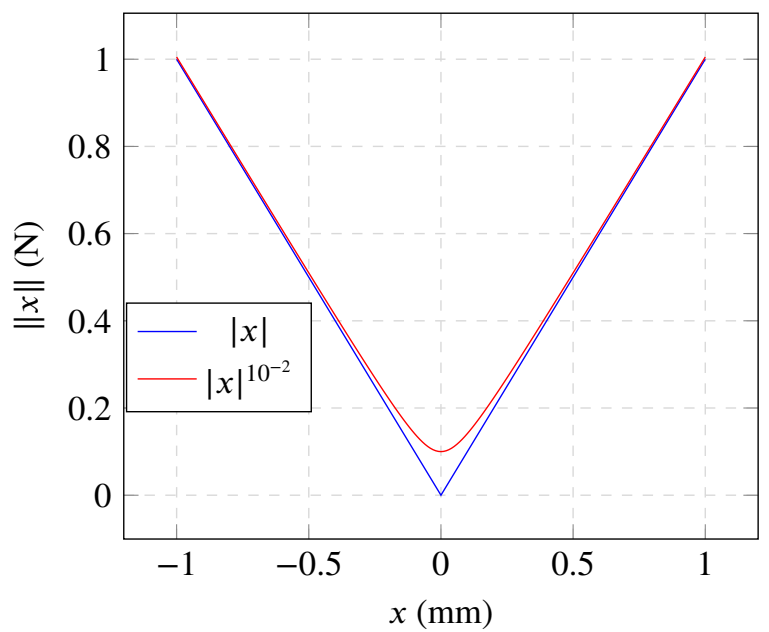

FIGURE 6 Comparison of the absolute value function and its smoothed version

The gradient of $N_{1}^{\epsilon}(\theta)$ is the following:

$$
\frac{\partial N_{1}^{\epsilon}(\theta)}{\partial \theta_{k}}=\frac{1}{2} \sum_{i} G_{i k} \frac{(G \theta)_{i}}{\sqrt{\left((G \theta)_{i}\right)^{2}+\epsilon}}
$$

Although being differentiable, $N_{1}^{\epsilon}$ has steep variations of its gradient. As a consequence, the convergence of the minimization algorithm is slower when this norm is used. The steps in the line-search tend to be smaller, and, what is much worse, the algorithm may get trapped in a local minimum. In order to ease the convergence, we propose to use a $\mathrm{N}_{2}$-regularized solution as the initialization $\theta_{0}$.

\section{3 | Choice of the parameters for the m-CRE method}

In many implementations of the m-CRE method, the regularization is ensured by hierarchical updating (only the zones with the highest error are corrected at each iteration) and by stopping the iterations at some point (see for example ${ }^{\sqrt{6}} \mathrm{or}^{23}$ ). In the present study, however, as the initial guess for the orientation is likely to be wrong everywhere, we prefer to allow the algorithm to update the whole parametric field at each iteration. This is why the regularization term $N(\theta)$ is added in order to add reliability 
to the field identification method. The drawback is that one has to tune two parameters, $\mu$ and $r$, instead of one. We then propose a way to a-priori choose a value for $r$, while $\mu$ will be determined thanks to a L-curve $\mathrm{e}^{24125}$.

We will use $\kappa$, the ratio of spectral radii of $K$ and $\Pi^{T} \Pi$. In practice, the computation of spectral radii is numerically costly, this is why in the numerical part, $\kappa$ will be replaced by $\tilde{\kappa}$, which is the ratio of Frobenius norms.

$\mathrm{In}{ }^{\sqrt[5]{5}}$, it appears that the main advantage of the m-CRE method is the ability to dispense with unreliable BCs. Numerical tests done in Section 5.2 tend to confirm this. As a consequence, we can choose for the weighting parameter $r$ any value that is small compared to $\kappa$. For example, $r=10^{-3} \kappa$. The method then tends towards PDE-constrained optimization, with the advantage of being able to ignore Dirichlet BCs. One can refer to Section 5.2.3. where the distance between the solution of m-CRE method (with BCs) and PDE-constrained optimization is numerically studied.

Another way to explain the choice of $r$ is by assuming that, as demonstrated in the context of time-harmonic elasticity in the theorem 2 of $\frac{16}{16}$, the higher is $r$, the more the problem is regularized. In the context of the proposed method, the regularization is rather performed by the term $N(\theta)$, and the role of $r$ is simply to provide a non-singular BC-free system. This is why any small value can be chosen, provided it ensures a correct condition number for the coupled problem 30 .

\section{5 | NUMERICAL RESULTS}

\section{1 | Boundary Conditions of the direct computation}

For the least-square minimization and standard m-CRE method of Sections 3.1 and 3.2 one needs to impose boundary conditions to the direct computation. It has been chosen to apply null displacements to the lower boundary (as it is blocked by the testing machine's jaw), to impose zero Neumann BCs on the lateral boundaries, and an homogeneous displacement on the upper boundary. The numerical value of the imposed vertical displacement is the mean of the measured displacement on this line, and the horizontal displacement is imposed equal to zero. These BCs are illustrated on Figure 7 In order to make the computed solution comparable with the measure, the operator $\Pi$ contains a projection that nullifies the rigid body motions.

For the m-CRE without unreliable Dirichlet BCs of Section 3.3 no Dirichlet BC is considered, as they tend to be uncertain, and the sides are considered to be free edges. For this variant, no special care has to be taken for the rigid body motions.

\section{2 | Comparison of different methods}

In this section, we aim at comparing the capabilities of the three different methods presented in Section 3 For that purpose, the regularization parameter $\mu$, which automatic determination is the purpose of part 5.4, is manually adjusted in order to obtain a visually satisfactory compromise between oscillation and resolution. The chosen values for $\mu$ are .1 when $N_{2}$ norm is used, and $10^{-4}$ for $N_{1}$ norm. 


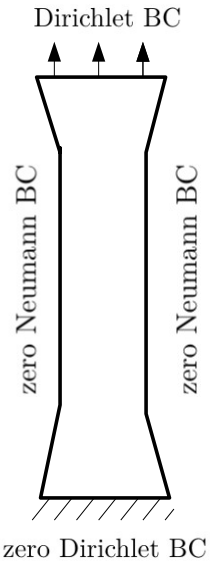

FIGURE 7 Boundary Conditions for the direct computation

\subsection{1 | Least-square minimization}

On Figure 8, one sees the result obtained with the least-square minimization method (Section 3.1). The regularizing norm is $N_{2}$. It can be noted that the identified trend is right (the fibers in the center of the specimen are more vertical), but the numerical values are quite false. The variations of the angles are over-estimated. Furthermore, extreme values appear in the corners. This is due to the fact that the corners are the points where the used BCs are the furthest from reality. The identified material parameters are the following: $E_{1}=154 G P a$ and $G_{12}=2.96 G P a$.

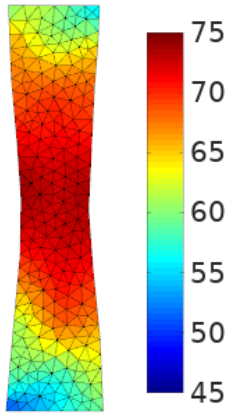

(a) Identification

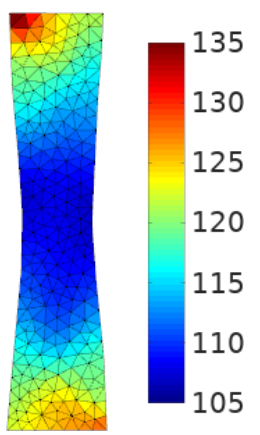

FIGURE 8 Identification by least-square method, $L^{2}$ regularization

\subsection{2 | m-CRE method}

In this section, the m-CRE method of Section 3.2 is evaluated on the experimental example. The $r$ parameter is settled to $\tilde{\kappa}$, the ratio of Frobenius norms of $K$ and $\Pi^{T} \Pi$. This means that the weight of the Constitutive Relation Error in the cost-function 
is roughly the same as the error in measurement. The quality of the identified field is comparable with the previous one, and, as previously, extreme values appear in the corners. The identified material parameters are the following: $E_{1}=92.6 G P a$ and $G_{12}=3.6 \mathrm{GPa}$.

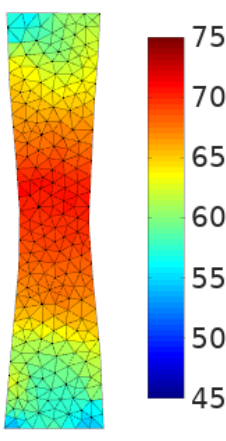

(a) Identification
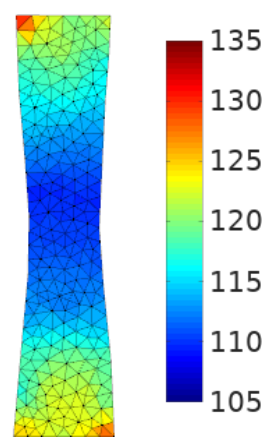

$\theta_{1}($ deg)

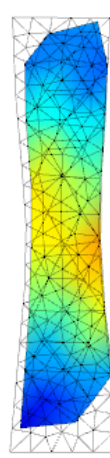

FIGURE 9 Identification by the m-CRE method, $L^{2}$ regularization

\subsection{3 | Link between least-square minimization and m-CRE}

The Euclidean distance between both vectors of parameters $\theta$ determined by least-square minimization and by m-CRE method is computed at convergence for different values of $r$. This distance is denoted by $d$ and is plotted on Figure 10

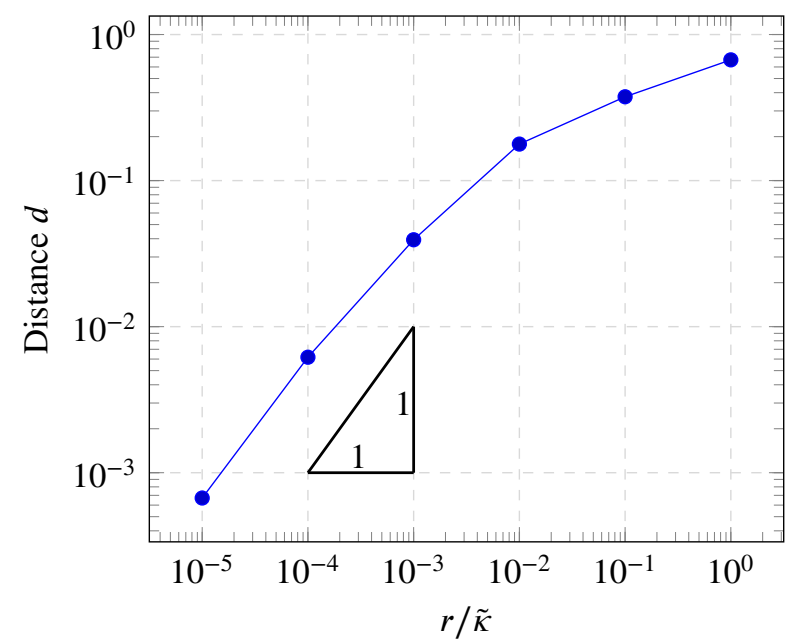

FIGURE 10 Euclidean distance between the solution of least-square minimization and m-CRE error, for different weighting parameters $r$ 
Wen $r / \tilde{\kappa}$ tends towards 0 , one observes that the distance also tends towards 0 , as predicted by the theory. What is more, the shape of the curve allows to intuit that this distance behaves like $O(r)$. For the rest of the article, we will fix $r=10^{-3} \tilde{\kappa}$ as this value leads to a solution that is sufficiently close to the least-square minimization solution, while ensuring that Equation 30 , has a good condition number in the case when some boundary conditions are ignored.

\subsection{4 | m-CRE method without BCs}

On Figure 11] one sees the identification performed with the m-CRE method with unknown BCs of Section 3.3 The BCs on the top and bottom edges are ignored. One observes a good agreement between the computed values and the measured ones. In particular, the artifacts in the corners have disappeared, which can be explained by the fact that we don't use Dirichlet BCs. What is more, the numerical values are way closer to the reference. The identified material parameters are the following: $E_{1}=142 G P a$ and $G_{12}=2.95 G P a$.

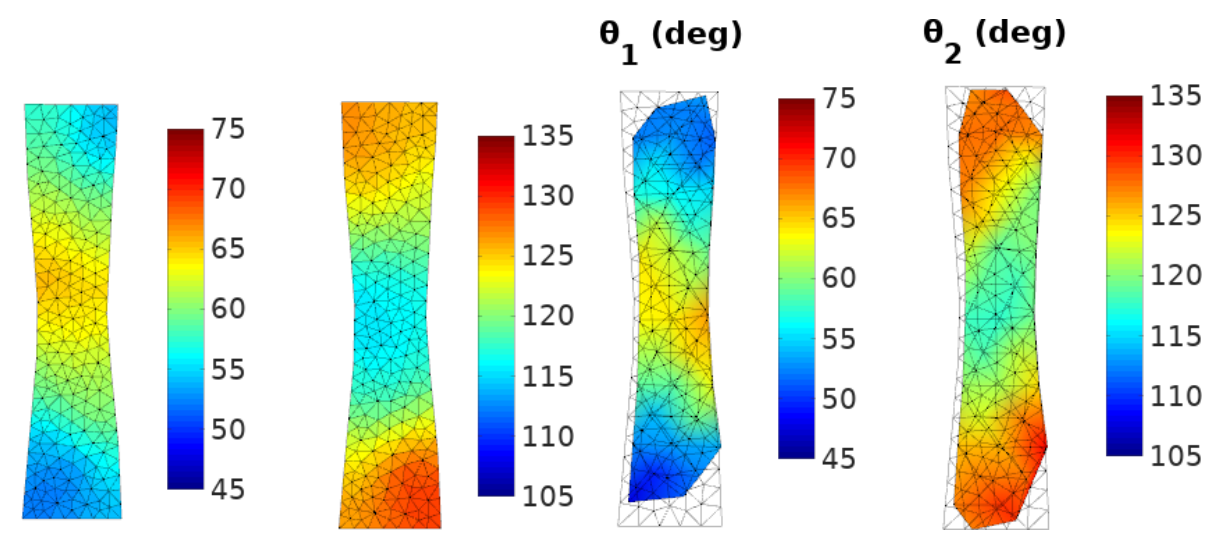

(a) Identification

(b) Reference

FIGURE 11 Identification by the m-CRE method, without Dirichlet BCs, $L^{2}$ regularization

On Figure 12, one sees the identification with a Total Variation regularized cost-function (compared to the reference field). This result was obtained by using the previous result to initialize the BFGS algorithm. This initialization ensures a safer convergence and is needed to overcome the difficulties caused by the Total Variation term. The identified values seem to be slightly better than on Figure 11 but the too coarse resolution of the reference field does not allow to definitely conclude about the rele-

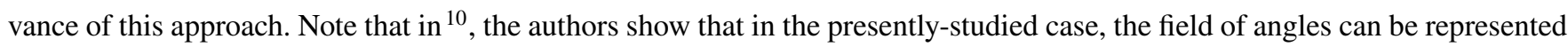
as uniform by parts. The identified material parameters are the following: $E_{1}=148 \mathrm{GPa}$ and $G_{12}=2,88 \mathrm{GPa}$. 


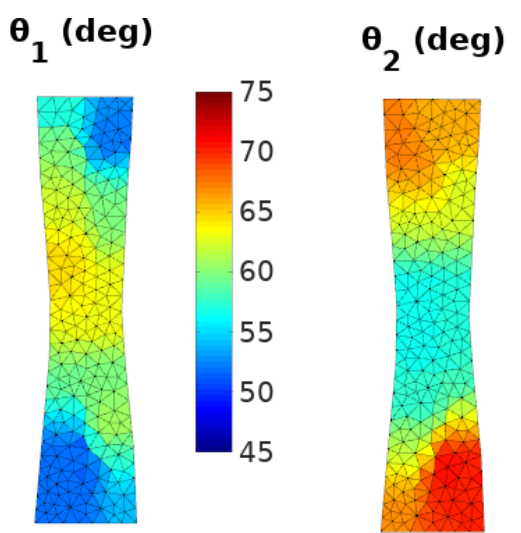

(a) Identification

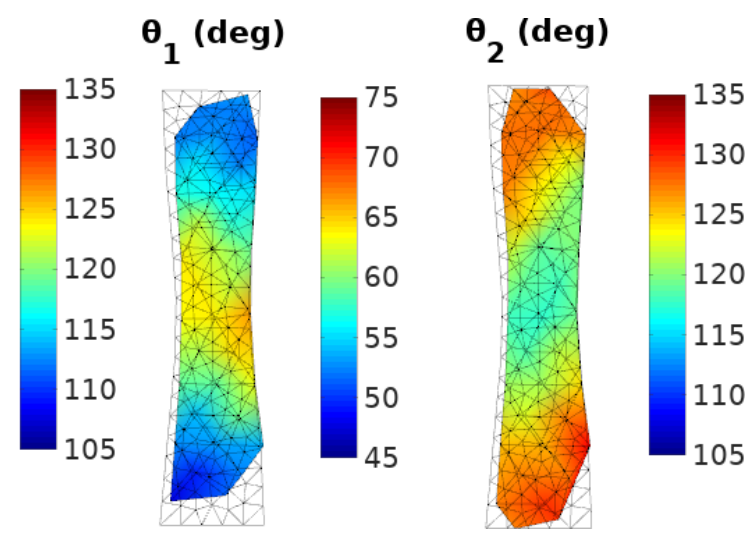

(b) Reference

FIGURE 12 Identification by the m-CRE method, without Dirichlet BCs, $L^{1}$ regularization

\section{3 | Evolution of the cost-function with respect to the parameters for m-CRE method without BCs}

The aim of this section is to empirically show the (mathematically not proven) convexity of the cost-function. The orientation fields and macroscopic parameters are fixed at the values obtained in Section 5.2.4 by the optimization method. Only one parameter will be changed at the time. We study the evolution of the two terms that constitute the cost-function, namely the residual and the regularizing term. The residual $R(u, v, \theta)$ is defined such that $R(u, v, \theta)+\mu r N(\theta)$ is the cost-function:

$$
R(u, v, \theta)=\frac{1}{2}(u-v)^{T} K(\theta)(u-v)+\frac{r}{2}\left(\Pi u-u_{m}\right)^{T}\left(\Pi u-u_{m}\right)
$$

Note that each evaluation of $R(\tilde{u}(\theta), \tilde{v}(\theta), \theta)$ requires to assemble the stiffness matrix $K(\theta)$ and solve the coupled problem (30) in order to obtain $\tilde{u}(\theta)$ and $\tilde{v}(\theta)$, as stated in Equation (30).

We first study the effect of the variation of the second angle $\left(\theta_{2}\right)$ at a gauss-point situated in the middle of the sample, as shown on Figure $13 \mathrm{a}$

Figure 13b displays the evolution of the residual with the parameter in question when the angle takes different values between the two "reasonable" values 105 and 135 degrees. One can observe that the variation of this residual is very small (although not strictly zero), and that it appears to be minimal for values smaller than 105 degrees, which is very far from the reference. This is a consequence of the ill-posedness of the un-regularized problem, and it shows that the residual alone is not enough to determine the angles at each Gauss point independently.

Figure 14 displays the evolution of the residual, the regularizing term and the total cost-function for the two different regularizing norms. The strong variations of the norm totally overwhelm those of the residual and one recognizes the quadratic shape for the norm $N_{2}$ and the linear-by-parts shape for the norm $N_{1}$. For both regularizing approaches, the solution is predominantly 


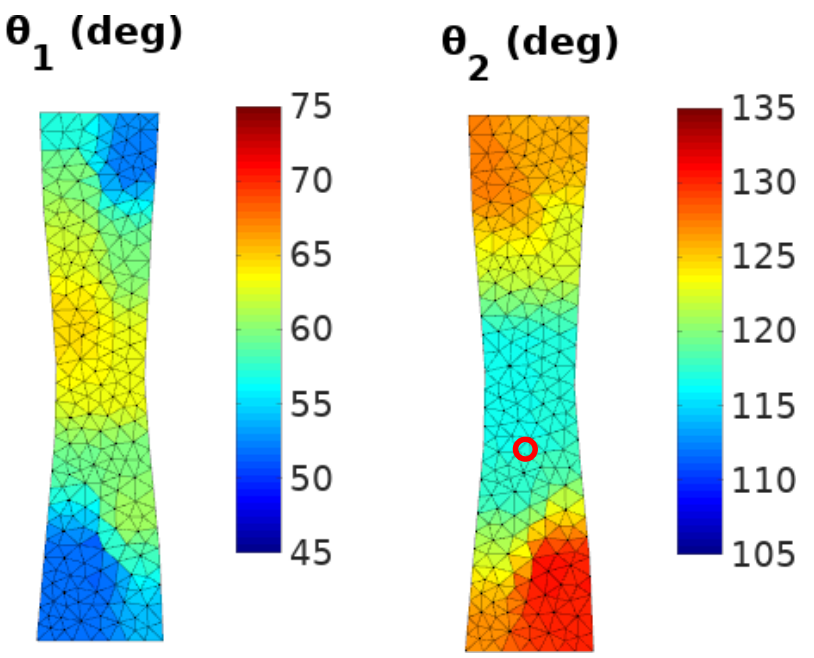

(a) Localization of the Gauss point on which the angle varies

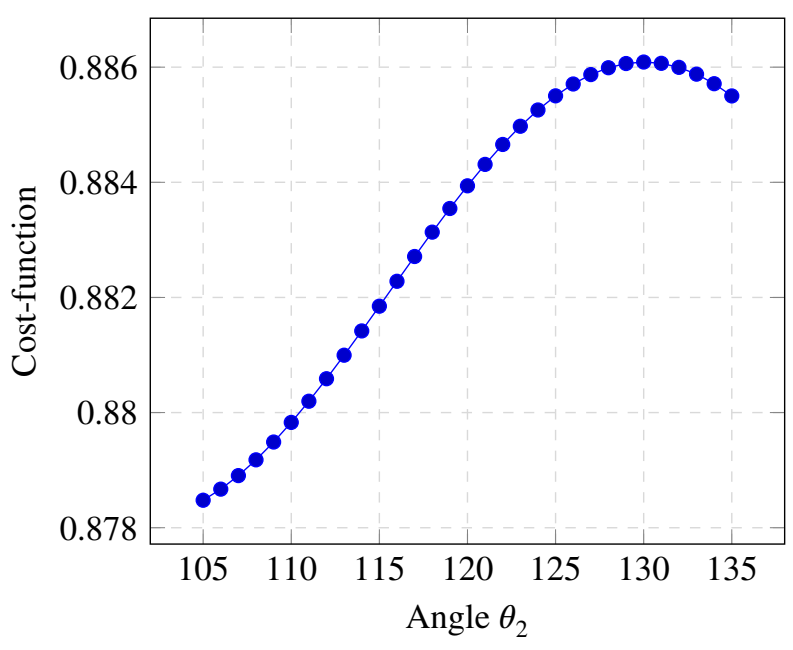

(b) Evolution of the Residual

FIGURE 13 Localization of the varying parameter and evolution of the residual

determined by the regularization term. This can be explained because only one parameter in the field varies independently of the others.

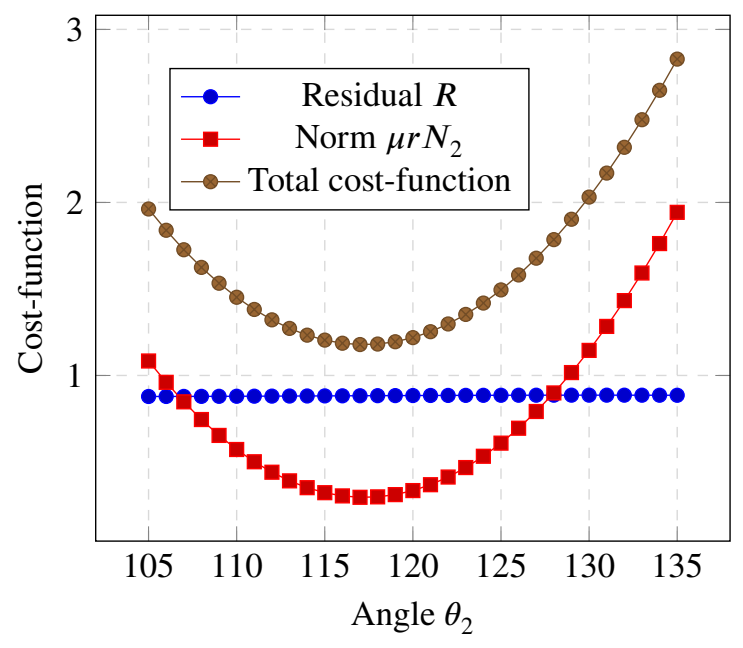

(a) $L^{2}$ with $\mu=.1$

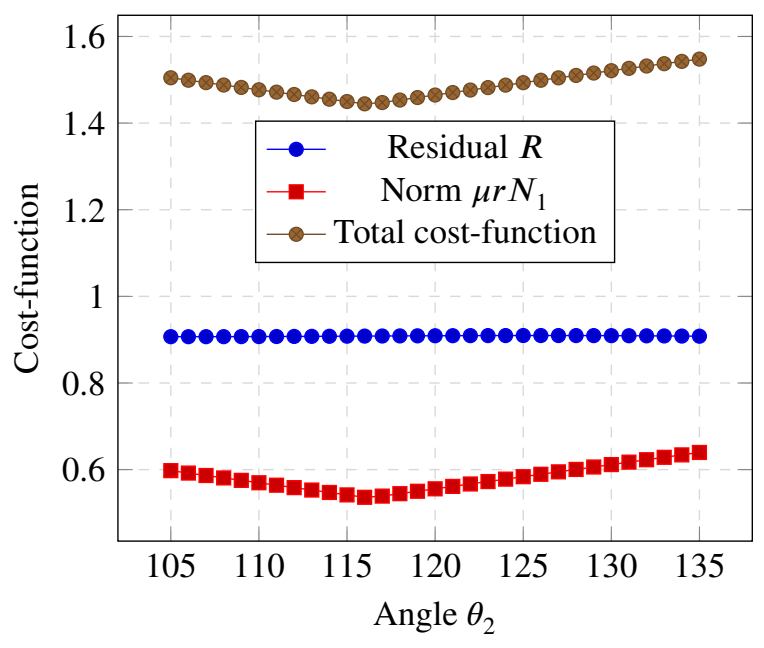

(b) $L^{1}$ (TV) with $\mu=10^{-4}$

FIGURE 14 Evolution of the residual, the regularizing term and the total cost-function with the angle $\theta_{2}$ on a particular point

Figure 15 displays the evolution of the same quantities when the Young modulus $E_{1}$ (which is a global parameter) varies around the value found in Section 5.2.4 denoted by $E_{1}^{\text {sol }}$. As the value of this modulus does not impact the norm $N_{2}$, the total cost-function is directly a shifted reproduction of the residual $R$. The result on this figure shows that, for this global parameter, the unregularized cost-function is convex and there is no need for extra regularization. 


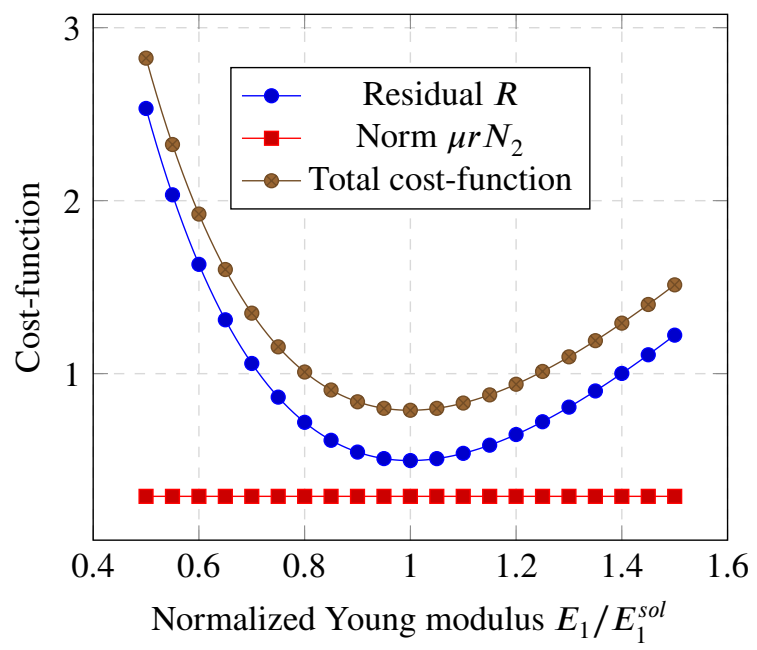

FIGURE 15 Evolution of the residual, the regularizing term and the total cost-function with the Young modulus

\subsection{Tuning of the regularization}

According to the results of Section 5.2, the most accurate method in the present case is the m-CRE with ignored unreliable BCs (of Section 3.3). As a consequence, all results in this part will be illustrated on this method.

\subsection{1 | Stopping iterates method}

In the application-problem, two separate groups of unknowns are identified, namely the angle fields and the material parameters. The regularizing norm $N(\theta)$ only stabilizes the angles. One could have added a second regularization term for the material parameters, but that would have required to introduce an other weighting parameter. That is why one chooses rather to stop the iterations in the case when the distance to initialization seems to increase dramatically. However, the case never occurred, so in practice, all numerical examples shown in this paper have been obtained without any regularization on the macroscopic material parameters.

The regularization via early-stopping of an iterative method has a strong theoretical background. It has for example been studied in ${ }^{26}$ in the context of linear ill-posed problems.

For the two orientation fields, the same idea of letting the number of iterates regularize the resolution has been tried. The parameter $\mu$ is set to zero, and the norm $N(\theta)$ is stored at every iteration. At the end of the run, a L-curve is plotted. This curve displays for every iteration the value of $R(u, v, \theta)$ against $N(\theta)$.

These L-curves are presented on Figure 16 for the norms $N_{2}$ and $N_{1}$ (corresponding respectively to the $L^{2}$ norm of the gradient, and the Total Variation of the field).

As proposed in $\frac{25}{}$, the stopping iterate is chosen by finding the corner (in sense of maximum curvature point) of the L-curve. On the test-case, both norms result in choosing iterate 3 as the optimal iterate, which is shown on Figure 17 This regularization 


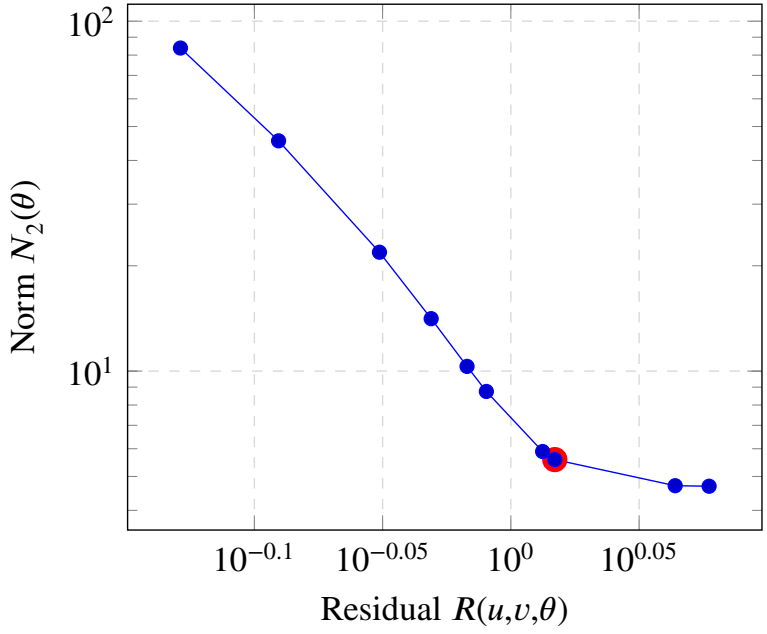

(a) $L^{2}$

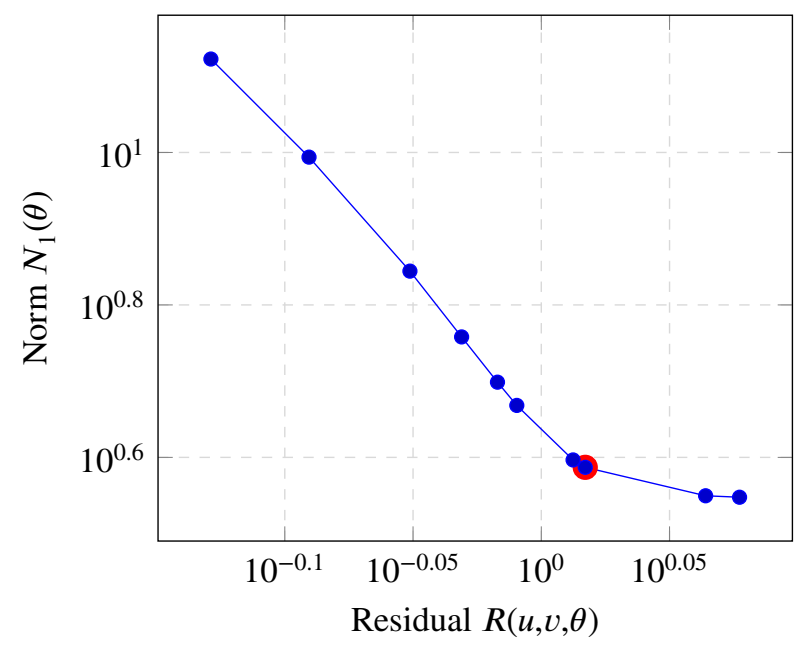

(b) $L^{1}(\mathrm{TV})$

FIGURE 16 L-curves for the stopping iterate regularization approach, with chosen iterate (red)

technique presents the advantage of being particularly simple as only one run of the algorithm is needed to determine the regularization parameter. What is more, it leads to a quite satisfactory result.

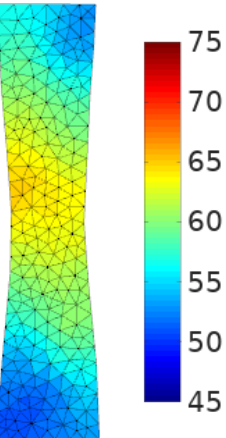

(a) Identification
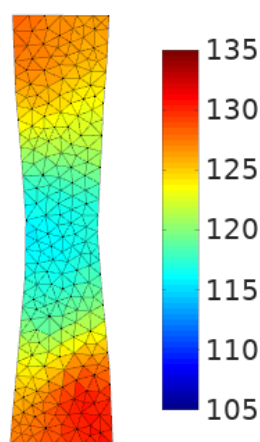

$\theta_{1}(\mathrm{deg})$

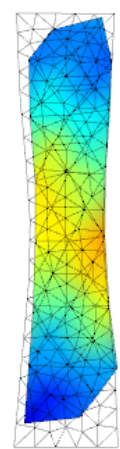

FIGURE 17 Identification by the m-CRE method, without Dirichlet BCs, 3rd iterate $\theta_{2}$ (deg)
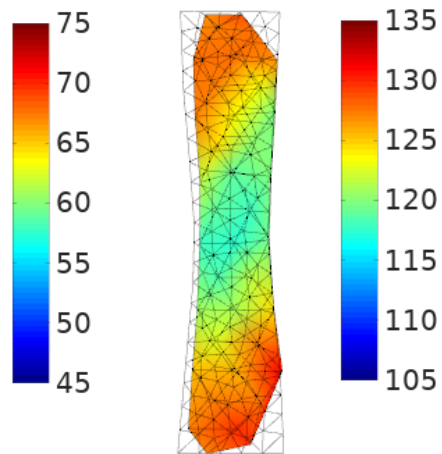

(b) Reference

25

20

15

10

05
However, the fact that both norms $N_{1}$ and $N_{2}$ lead to the same solution shows that this regularization technique is not very flexible. This is why, in the following subsection, another approach is investigated. 


\subsection{2 | Tikhonov method}

With this approach, and contrary to the stopping iterate method, the iterative minimization algorithm will be carried out until convergence of the cost-function, and the regularization will come from the term $N(\theta)$, and its weight, $\mu$. The key question is to automatically identify a suitable value for this weight.

One manually identifies a "viable" interval of variation where the fields are not visually uniform, nor presenting high oscillations. This interval is sampled, and we perform the inversion for several values of $\mu$. A L-curve is then plotted, that presents the norm $N(\theta)$ in ordinate and the residual $R(u, v, \theta)$ in abscissa for all values of $\mu$. This curve is available on Figure 18

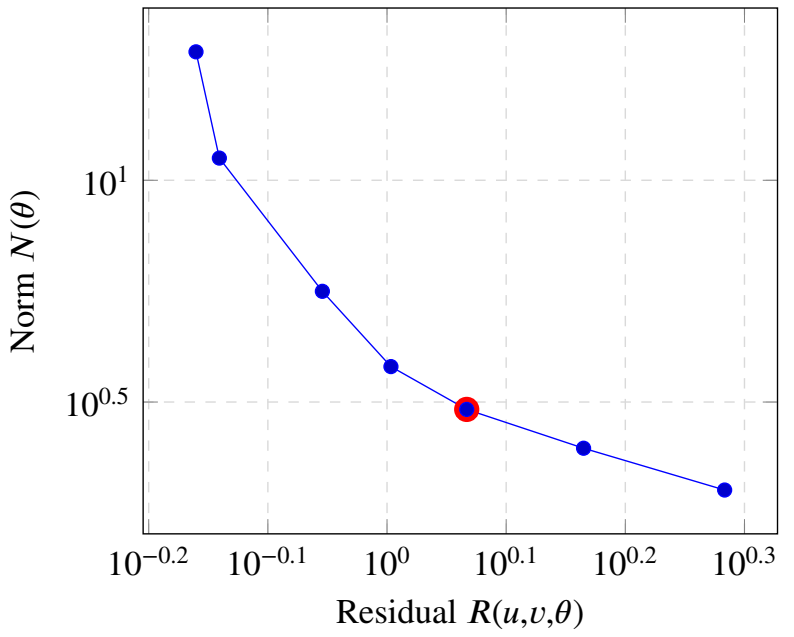

(a) $L^{2}$

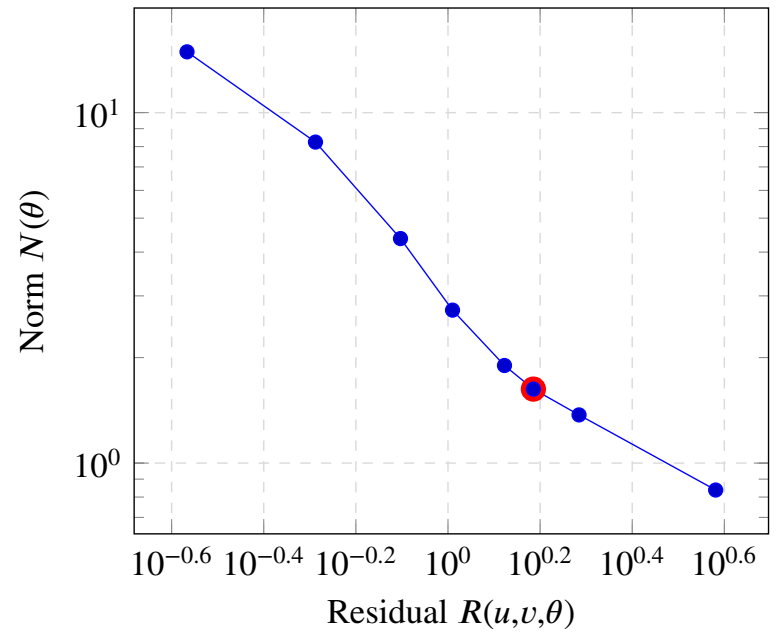

(b) $L^{1}(\mathrm{TV})$

FIGURE 18 L-curves for the Tikhonov regularizing approach, with the chosen setting (red)

The first remark is that the L-curve for the $L^{2}$ norm has the expected concave shape, but its corner is not well marked. On the other hand, the shape of the L-curve for the $L^{1}$ norm seems to be less favourable, but its corner is more visible. Two methods for the automatic detection of this corner have been tested. The first one is inspired from ${ }^{[25}$ and consists in finding the maximum curvature point of the L-curve. The curvature has been computed from the first and second derivatives of the parametrized Lcurve, obtained by finite differences. The second method is described in 27 . It consists in finding the point along the L-curve that minimizes the product $R(u, v, \theta) \times N(\theta)$. This method is easier to apply as there is no need to analyse the shape of the L-curve, but one could probably find out applications where it leads to an aberrant solution.

By chance, on the two presented examples, the optimal points in the L-curves obtained by the two methods are the same (marked in red on the figure 18). They are of course expected to be close, but in cases when the shape of the L-curve is more complex, differences may appear between the two methods, as studied in 28 . 
When the L-curve is smooth, as it is the case here, it is possible to create a quadratic interpolation of the curvature around the maximum curvature point in order to find an optimal value of $\mu$ in the interval for which the interpolation has been performed. By this means, we obtain $\mu=.16$ with the norm $N_{2}$ and $\mu=7.9 \cdot 10^{-4}$ with the norm $N_{1}$.

Figure 19 presents the result of the identification process with the norm $N_{2}$ and regularization parameter $\mu=.16$. This result is good and close to the one shown on Figure 11 This is not surprising as the regularization parameters used for both approaches are close.

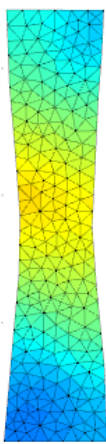

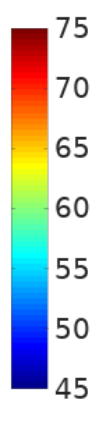

(a) Identification $\theta_{1}(\operatorname{deg})$

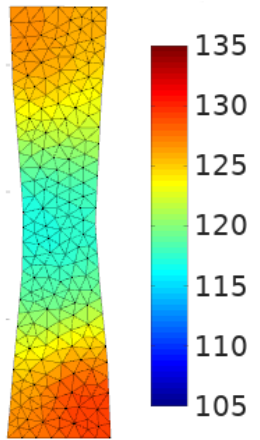

FIGURE 19 Identification with the $N_{2}$ norm, $\mu=.16$ $\theta_{2}(\mathrm{deg})$
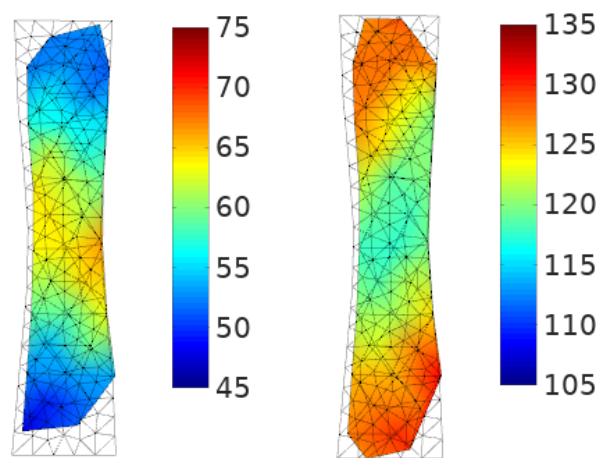

(b) Reference

Figure 20 presents the result of the identification process with the norm $N_{1}$ and regularization parameter $\mu=7.9 \cdot 10^{-4}$. This result seems to be over-regularized. In this context, the automatic determination of the regularization parameter seems to fail to select the best value. The result shown on Figure 12 , with $\mu=10^{-4}$ is of way better quality.

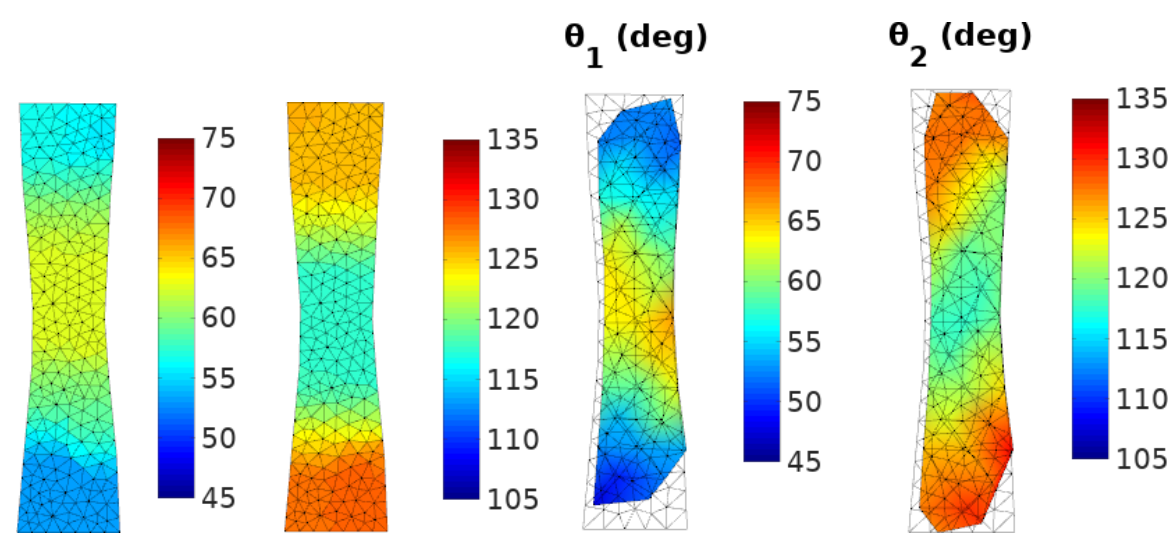

(a) Identification

(b) Reference

FIGURE 20 Identification with the $N_{1}$ norm, $\mu=7.9 \cdot 10^{-4}$ 
Another method for choosing the regularization parameter is the Morozov principle 29 , that presents the advantage of using the information of the noise level on the measurement, and thus not to fall under the Bakushinkii veto 30 . When applied to the m-CRE method, the Morozov principle consists in computing an expected value of the measurement mismatch $\mathfrak{m}$, from the standard deviation on the measurement $\sigma$, obtained in Section 2.2. $\mathfrak{m}=\sigma^{T} \sigma$. We choose, among all values that have been tested for $\mu$, the largest one that ensures $\left(\Pi u-u_{m}\right)^{T}\left(\Pi u-u_{m}\right) \leqslant \mathfrak{m}$. In the present case, the Morozov principle would lead to $\mu=.1$ with the norm $N_{2}$ and $\mu=5 \cdot 10^{-4}$ with the norm $N_{1}$. These results are close to those obtained by the L-curve method. This confirms the coherence of the proposed methods for the choice of $\mu$.

All the values for $\mu$ obtained with the three studied methods and for the two proposed regularizing terms are summarized in Table 1

\begin{tabular}{|c|c|c|c|}
\hline Regularizing norm & $\mu$ maximum curvature & $\mu$ minimum product & $\mu$ Morozov \\
\hline$L^{1}$ & $7.9 \cdot 10^{-4}$ & $10^{-3}$ & $5 \cdot 10^{-4}$ \\
\hline$L^{2}$ & .16 & .1 & .1 \\
\hline
\end{tabular}

TABLE 1 Chosen values for $\mu$ depending on the choice method

To conclude, in this numerical experiment, the different methods that have been tested to automatically determinate the regularization parameter have given successful and coherent results when the norm $N_{2}$ is used. In the case of the norm $N_{1}$, the results appear to be poorer. A possible explanation for this could have been that, due to the lack of utilization of a dedicated algorithm, the $L^{1}$-regularized cost-function optimization is inefficient. However, a numerical experimentation with the split Bregman algorithm from ${ }^{22]}$ led to the same outcome.

This is what led us to formulate another hypothesis: The $L^{1}$ regularization shows poorer results because the test-case that has been studied until now presents rather smooth variations of the parametric field on the whole domain. For this kind of distribution, it can be expected that the $L^{2}$ norm is better suited to identification.

\subsection{3 | Study of another test-case}

We propose in this section another test-case for which the $L^{1}$ norm is expected to give better results. The reference angles are presented on Figure 21. This sample has been subjected to a higher pre-polymerization deformation, which results in a thinner shape and a more homogeneous orientation field. The only zones where the orientations are different from the rest of the sample are close to the extremities. As a consequence, the problem is closer to a defect identification problem.

Like before, we plot on Figure 22 the L-curves for both regularizing norms. The algorithm that searches for the maximum curvature obviously finds a point on both curves. However, one has to notice that the L-curve in the $L^{2}$ case is very flat, which 


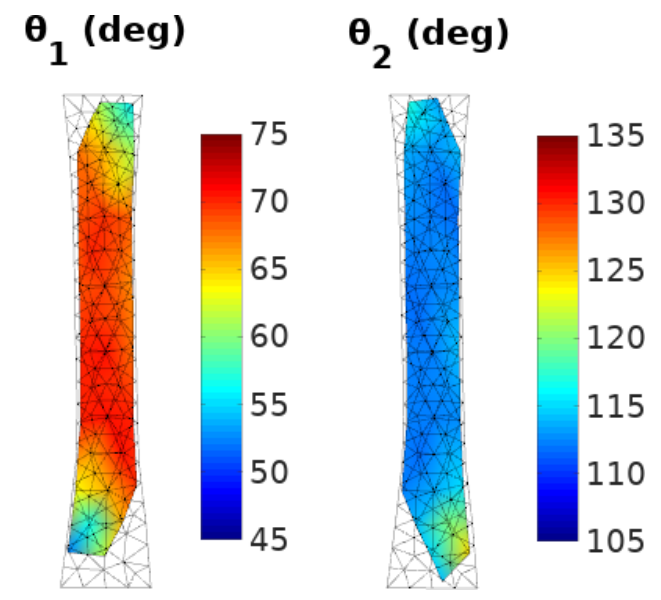

FIGURE 21 Measured angles on the second specimen

makes its "corner" not really relevant. On the other hand, the curve in the $L^{1}$ case has a clear slope break, even if it is not very strong. The optimal values obtained are 0.114 in the $L^{2}$ case and $3.4984 \cdot 10^{-4}$ in the $L^{1}$ case.

Furthermore, the minimum product criterion points once again towards the same value as the maximum curvature criterion in the $L^{1}$ case: $\mu=5 \cdot 10^{-4}$. On the contrary, in the $L^{2}$ case, this minimum product criterion points towards the highest possible value of the regularization parameter $(\mu=1)$, and gives thus a very different result from the maximum curvature criterion.

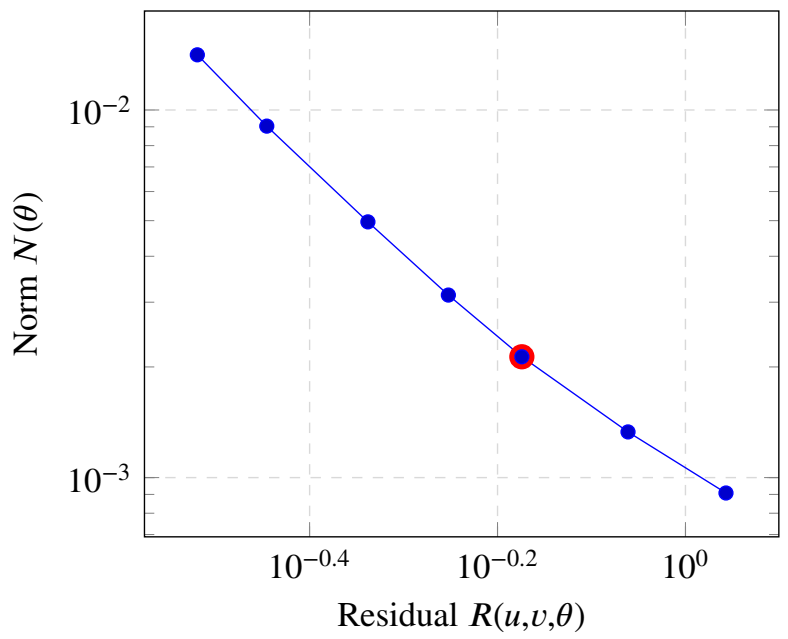

(a) $L^{2}$

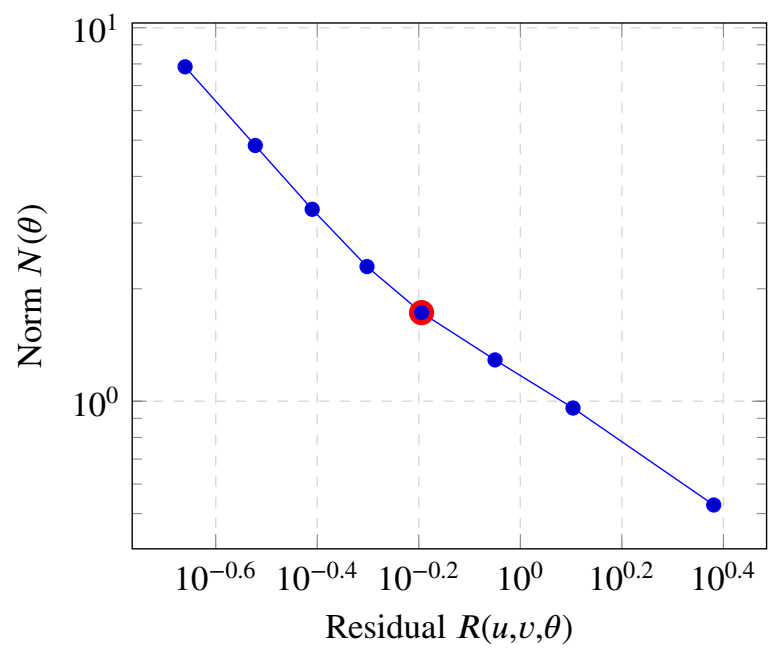

(b) $L^{1}(\mathrm{TV})$

FIGURE 22 L-curves for the Tikhonov regularizing approach, second test-case, with the maximum curvature point (red)

Finally, the Morozov principle leads to $\mu=.02$ in the $L^{2}$ case and $\mu=1 \cdot 10^{-4}$ in the $L^{1}$ case.

To sum up on the choice of the regularization parameter, all the values for $\mu$ obtained with the three studied methods and for the two proposed regularizing terms are displayed in Table 2. It appears that for the $L^{2}$ regularization, the three methods 
give very different results. On the other hand, for the $L^{1}$ regularization, the three methods lead to values that, although being noticeably different, have the same order of magnitude.

\begin{tabular}{|c|c|c|c|}
\hline Regularizing norm & $\mu$ maximum curvature & $\mu$ minimum product & $\mu$ Morozov \\
\hline$L^{1}$ & $3.4984 \cdot 10^{-4}$ & $5 \cdot 10^{-4}$ & $10^{-4}$ \\
\hline$L^{2}$ & .114 & 1 (highest value tested) & .02 \\
\hline
\end{tabular}

TABLE 2 Chosen values for $\mu$ depending on the choice method

On Figures 23 and 24 we compare the identified orientation fields with both $L^{2}$ and $L^{1}$ regularization. As all three parameter choice method have given different results on the $L^{2}$ case, it was decided to use the Morozov principle, which offers the advantage to use the knowledge of the uncertainty on the measurement.

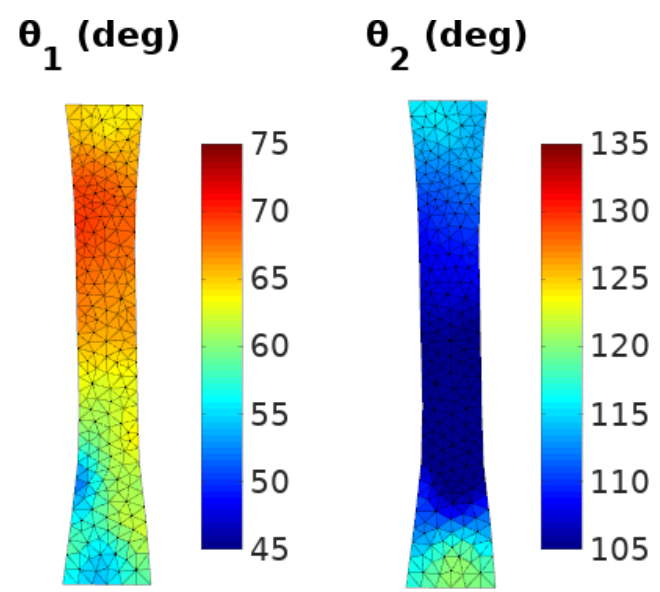

(a) Identification

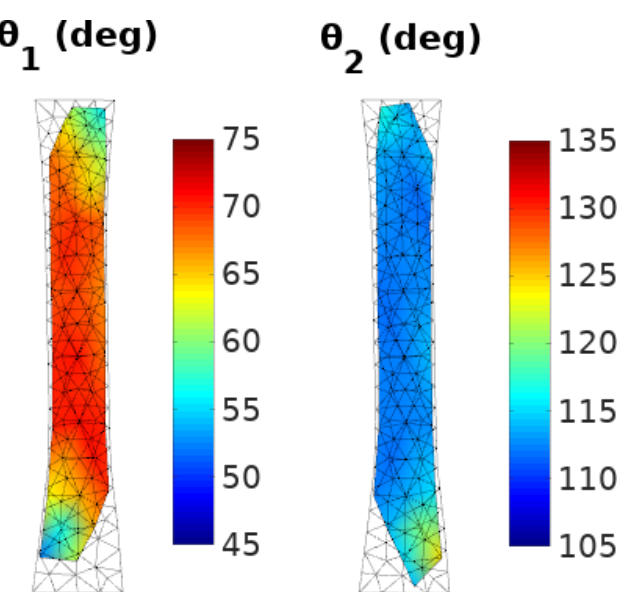

(b) Reference

FIGURE 23 Identification with the $N_{2}$ norm, $\mu=0.02$

The field identified with $L^{2}$ regularization term shows the correct trend for the identified values of the angles. However, it presents an unwanted gradient of angle in the central zone of the sample, where it should be homogeneous.

The field identified with $L^{1}$ regularization, on the other hand, is close to homogeneous by parts. The angle variations in the bottom part of the sample are detected without any addition of un-relevant gradients elsewhere. One should however mention that the much smaller angle variation in the top part of the sample remains mostly undetected, and the mean value of $\theta_{2}$ in the central zone is wrong by about 5 degrees.

Despite those two misfits, the $L^{1}$ regularization method appears here to be the superior method for two reasons: firstly, the three parameter choice methods give coherent results, and secondly, the shape of the identified field is more accurate. 


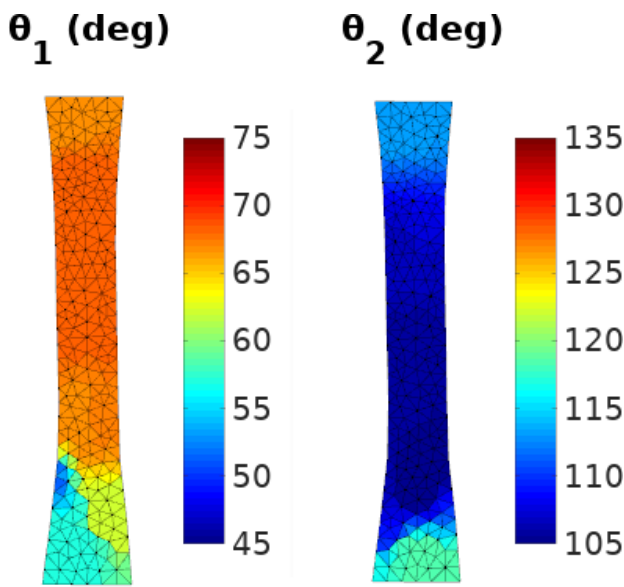

(a) Identification $\theta_{1}(\operatorname{deg}) \quad \theta_{2}(\mathrm{deg})$

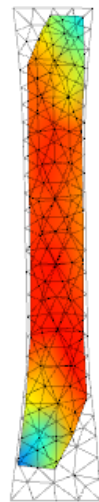

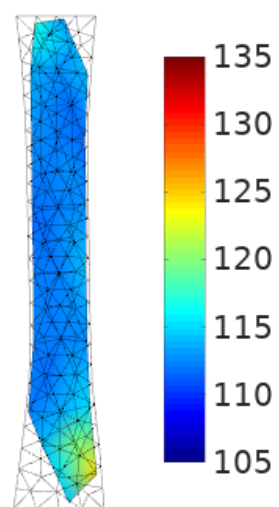

(b) Reference

FIGURE 24 Identification with the $N_{1}$ norm, $\mu=1 \cdot 10^{-4}$

\subsubsection{Impact of the discretization of $\theta$}

One can expect the choice of the discretization of the fields $\theta_{1}$ and $\theta_{2}$ to have a significant influence on their identification. In order to investigate this aspect, we create clusters of adjacent elements on which the values of the fields are imposed to be homogeneous, as presented on Figure 25a This has the effect of reducing the number of unknowns, and thus the cost of each resolution of the linear system 36 . The m-CRE identification is then run on this discretization in order to identify the fields displayed on Figure 25. The identified field appears to be in agreement with the field displayed on Figure 19, but with a lower resolution due to the lower number of unknowns.

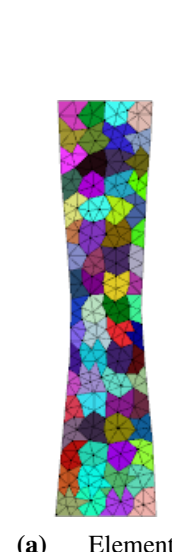

clusters
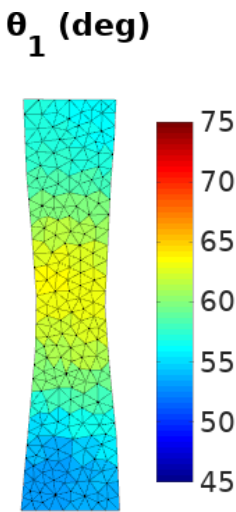

(b) Identification $\theta_{2}(\operatorname{deg})$
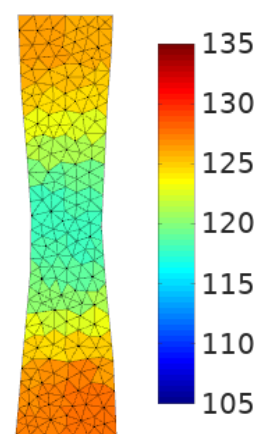

105

$\theta_{1}$ (deg)
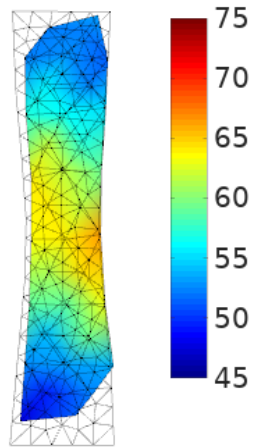

(c) Reference $\theta_{2}($ deg)

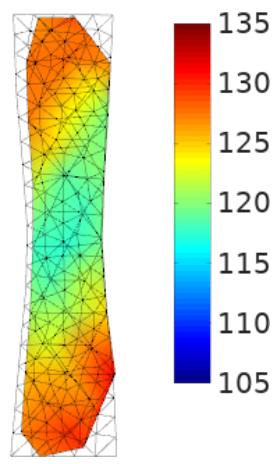

130

125

120

10

05

FIGURE 25 Identification with norm $N_{2}, \mu=0.16$ and spacial coupling of elements

The fact that the elements are clustered adds a kind of spacial regularization to the minimization. Figure 26]displays the result of the identification in the case where the parameter $\mu$ is set to 0 and the spacial regularization is only ensured by the clustering 
of the elements. It can be noted that the result is far from being satisfactory. In addition, we had to impose the global parameters $E_{1}$ and $G_{12}$ to be equal to the values found in Section 5.2.4 in order to stabilize the resolution.

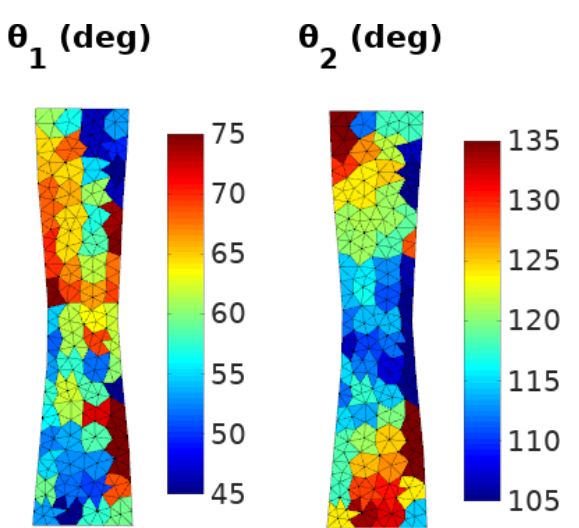

(a) Identification $\theta_{1}(\mathrm{deg})$

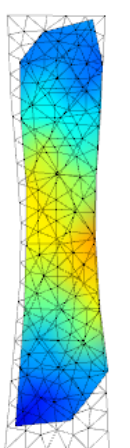

$\theta_{2}($ deg)
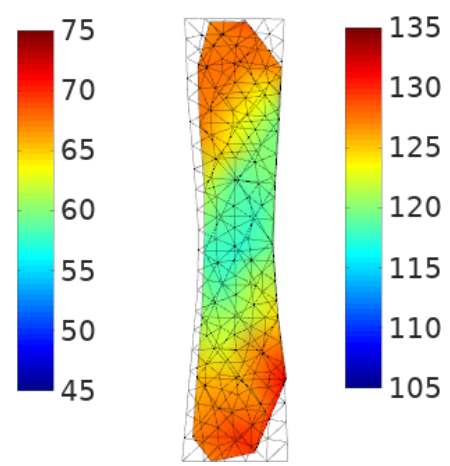

(b) Reference

FIGURE 26 Identification with $\mu=0$ and spacial coupling of elements

On Figure 27, the same idea is tried with larger clusters, but the result is still not satisfactory. The conclusion of this numerical experiment is that the adoption of a gradient-based regularization term gives much better results than the clustering of elements used in this section.

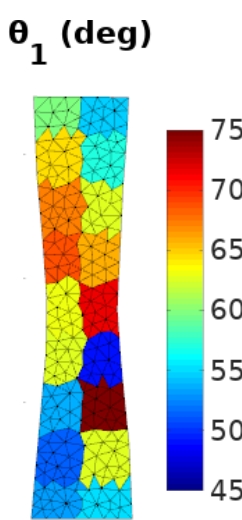

(a) Identification

$$
\theta_{2}(\mathrm{deg})
$$
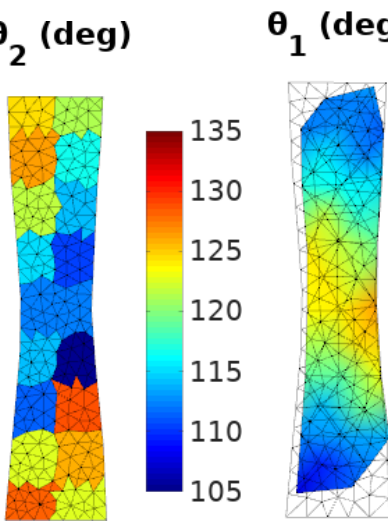

FIGURE 27 Identification with $\mu=0$ and spacial coupling of elements (bigger clusters) 


\section{6 | CONCLUSION}

In this study, two variants of the modified Constitutive Relation Error method have been studied both theoretically and numerically, and compared to another classical method, namely the PDE-constrained optimization, on an experimental test-case.

All theoretical developments done in this paper have been confronted with experimental measurements. The test-problem consists in the identification of the inhomogeneous orientation of the fibers of a composite specimen from full-field displacement measurement. As the global stiffness parameters of the studied material are only roughly known, this experiment enabled us to test the identification of a few global parameters along with the parametric fields. Such an identification method has proven to converge well.

It was shown that the use of a norm of the gradient of the identified field is an interesting way to enhance the quality of the identification with the m-CRE method. A term proportional to this norm can either be directly added to the function that is minimized, or it can serve as an ingredient of a regularizing stopping criterion. Two norms of the gradient have been tested, in order to illustrate the fact that while the $L^{2}$-regularized solution is smooth on the whole domain, the $L^{1}$-regularized solution tends to have rather homogeneous zones, separated by sharp frontiers. The choice between $L^{1}$ and $L^{2}$ norms is meant to be made from the a priori knowledge of the shape of the identified field.

The automatic determination of the two parameters of the method is a critical point in the perspective of designing blackbox tools for identification. For this purpose, we proposed to impose the weighting parameter to take an arbitrary value that is small compared to a particular ratio of spectral radii. In that case, the proposed method can then simply be interpreted as a generalization of the least-square minimization method in order not to be forced to use unreliable BCs on the domain. For the determination of the regularization parameter, both L-curve and Morozov method have been tested.

Two different test-cases have been investigated. The $L^{2}$-regularized cost-function seems better suited to the first one, for which the field to identify has smooth gradients. The second test-case presents a large homogeneous zone and sharp variations of the field at its extremities and turns out to be overall more challenging. It seems to be better handled by the $L^{1}$-regularized cost-function. This investigation highlights the fact that the two proposed regularization terms are best applied to different test-cases.

Two main perspectives emerge from this study. The first one concerns the application of the method to dynamics problems, that are known to be more opportune for the identification, in order to identify a full heterogeneous field of orthotropic stiffness parameters of the material. The second perspective is the acceleration of the computation. The fact that many similar direct resolutions have to be performed in order to identify the parameters has led many authors to propose the use of a reduced order model, like in ${ }^{31}$. In our case, the reduction method has to be able to deal with a field of parameters, as does the method presented in 32 . 


\section{DATA AVAILABILITY STATEMENT}

The data that support the findings of this study are available from the corresponding author upon reasonable request.

\section{REFERENCES}

\section{References}

1. Avril S, Bonnet M, Bretelle AS, et al. Overview of identification methods of mechanical parameters based on full-field measurements. Experimental Mechanics 2008; 48(4): 381.

2. Ladeveze P, Reynier M, Nedjar D. Parametric correction of finite element models using modal tests. In: Springer. 1993 (pp. 91-100).

3. Allix O, Feissel P, Thévenet P. A delay damage mesomodel of laminates under dynamic loading: basic aspects and identification issues. Computers \& structures 2003; 81(12): 1177-1191.

4. Bonnet M, Aquino W. Three-dimensional transient elastodynamic inversion using an error in constitutive relation functional. Inverse Problems 2015; 31(3): 035010.

5. Ben Azzouna M, Feissel P, Villon P. Robust identification of elastic properties using the modified constitutive relation error. Computer Methods in Applied Mechanics and Engineering 2015; 295: 196-218.

6. Barbarella E, Allix O, Daghia F, Lamon J, Jollivet T. A new inverse approach for the localization and characterization of defects based on compressive experiments. Computational Mechanics 2016; 57(6): 1061-1074.

7. Seidl DT, Oberai AA, Barbone PE. The Coupled Adjoint-State Equation in forward and inverse linear elasticity: Incompressible plane stress. Computer Methods in Applied Mechanics and Engineering 2019; 357: 112588.

8. Hansen PC. Regularization, GSVD and truncated GSVD. BIT Numerical Mathematics 1989; 29(3): 491-504.

9. Phillips DL. A technique for the numerical solution of certain integral equations of the first kind. Journal of the ACM (JACM) 1962; 9(1): 84-97.

10. Launay J, Hivet G, Duong AV, Boisse P. Experimental analysis of the influence of tensions on in plane shear behaviour of woven composite reinforcements. Composites science and technology 2008; 68(2): 506-515.

11. Jauffret M, Cocchi A, Naouar N, Hochard C, Boisse P. Textile Composite Damage Analysis Taking into Account the Forming Process. Materials 2020; 13(23): 5337. 
12. ARAMIS . http : //www.gom.com. GOM . 2015.

13. Gay D. Composite materials: design and applications. CRC press . 2014.

14. Banerjee B, Walsh TF, Aquino W, Bonnet M. Large scale parameter estimation problems in frequency-domain elastodynamics using an error in constitutive equation functional. Computer methods in applied mechanics and engineering 2013; 253: $60-72$.

15. Guo J, Wang L, Takewaki I. Frequency response-based damage identification in frames by minimum constitutive relation error and sparse regularization. Journal of Sound and Vibration 2019; 443: 270-292.

16. Aquino W, Bonnet M. Analysis of the error in constitutive equation approach for time-harmonic elasticity imaging. SIAM Journal on Applied Mathematics 2019; 79(3): 822-849.

17. Caillé L, Hanus J, Delvare F, Michaux-Leblond N. MFS fading regularization method for the identification of boundary conditions from partial elastic displacement field data. European Journal of Computational Mechanics 2018; 27(5-6): 508539.

18. Fletcher R. A new approach to variable metric algorithms. The computer journal 1970; 13(3): 317-322.

19. Chan TF, Golub GH, Mulet P. A nonlinear primal-dual method for total variation-based image restoration. SIAM journal on scientific computing 1999; 20(6): 1964-1977.

20. Mascareñas D, Cattaneo A, Theiler J, Farrar C. Compressed sensing techniques for detecting damage in structures. Structural Health Monitoring 2013; 12(4): 325-338.

21. Zhang Z, Xu Y, Yang J, Li X, Zhang D. A survey of sparse representation: algorithms and applications. IEEE access 2015; 3: $490-530$.

22. Goldstein T, Osher S. The split Bregman method for L1-regularized problems. SIAM journal on imaging sciences 2009; 2(2): $323-343$.

23. Chamoin L, Ladevèze $\mathrm{P}$, Waeytens J. Goal-oriented updating of mechanical models using the adjoint framework. Computational mechanics 2014; 54(6): 1415-1430.

24. Miller K. Least squares methods for ill-posed problems with a prescribed bound. SIAM Journal on Mathematical Analysis 1970; 1(1): 52-74.

25. Hansen PC. Analysis of discrete ill-posed problems by means of the L-curve. SIAM review 1992; 34(4): 561-580. 
26. Santos RJ. Equivalence of regularization and truncated iteration for general ill-posed problems. Linear algebra and its applications 1996; 236: 25-33.

27. Regińska T. A regularization parameter in discrete ill-posed problems. SIAM Journal on Scientific Computing 1996; 17(3): 740-749.

28. Johnston PR, Gulrajani RM. Selecting the corner in the L-curve approach to Tikhonov regularization. IEEE Transactions on biomedical engineering 2000; 47(9): 1293-1296.

29. Morozov VA. The error principle in the solution of operational equations by the regularization method. Zhurnal Vychislitel'noi Matematiki i Matematicheskoi Fiziki 1968; 8(2): 295-309.

30. Bakushinskii A. Remarks on choosing a regularization parameter using the quasioptimality and ratio criterion. USSR Computational Mathematics and Mathematical Physics 1985; 24(4): 181-182.

31. Marchand B, Chamoin L, Rey C. Real-time updating of structural mechanics models using Kalman filtering, modified constitutive relation error, and proper generalized decomposition. International Journal for Numerical Methods in Engineering 2016; 107(9): 786-810.

32. Paillet C, Néron D, Ladevèze P. A door to model reduction in high-dimensional parameter space. Comptes Rendus Mécanique 2018; 346(7): 524-531. 